Supporting Information:

\title{
Synthesis, Structure, and LLCT Transitions in \\ Terminal Hydrazido(2-) Bipyridine Complexes
}

\section{of Titanium}

\author{
Sameer Patel, Yahong Li, ${ }^{\dagger}$ Aaron L. Odom* \\ Michigan State University, Department of Chemistry, East Lansing, MI 48824. \\ Email:odom@cem.msu.edu
}

A. Structure of Ti[NN(H)Ph][dpma][Bu ${ }^{t}$-bpy] (8) from x-ray diffraction

B. Discussion on modeling the electron transfer, Gaussian Fits, and fitting parameters for complexes $5-8$.................................................................................................................... $S 9$

C. Tables of $\Delta E^{o}$ calculations for complexes 5-8 ...................................................S28

D. Plots of $E_{o p}-\Delta E^{\circ}$ versus $1 / D_{o p}-1 / D_{s}$ for complexes 6-8...........................................S29

E. Solvent dielectric constant vs $E_{\text {op }}$ plots for complexes 2-8.....................................S31

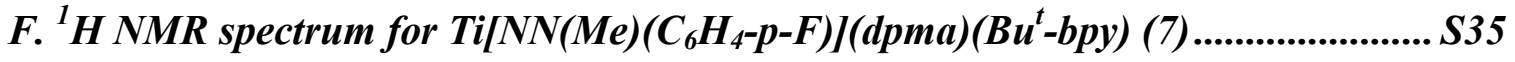

G. ${ }^{1} \mathrm{H}$ NMR spectrum for Ti[NN(H)Ph](dpma) $\left(B u^{t}-b p y\right)(8)$..................................... S35 
A. Structure of $\mathrm{Ti}[\mathrm{NN}(\mathrm{H}) \mathrm{Ph}][\mathrm{dpma}]\left[\mathrm{Bu}^{\mathrm{t}}-\mathrm{bpy}\right](\mathrm{8})$ from $\mathrm{x}$-ray diffraction
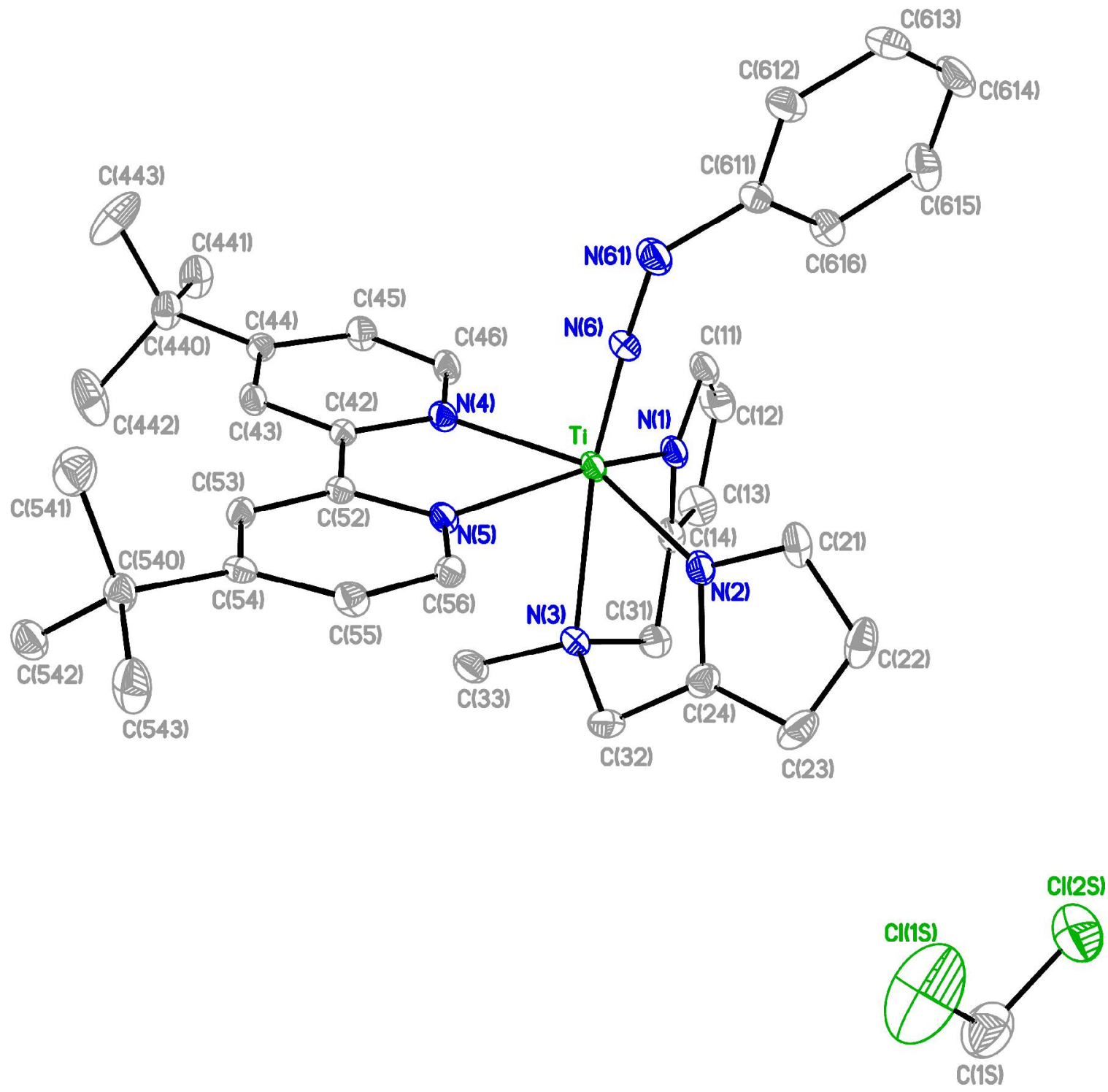
Table 1. Crystal data and structure refinement for Ti [NN(H) Ph] [Bu ${ }^{\mathrm{t}}-\mathrm{bpy}$ [dpma] .

Identification code

Empirical formula

Formula weight

Temperature

Wavelength

Crystal system, space group

Unit cell dimensions

Volume

Z, Calculated density

Absorption coefficient

$F(000)$

Crystal size

Theta range for data collection

Limiting indices

Reflections collected / unique

Completeness to theta $=23.39$

Absorption correction

Max. and min. transmission

Refinement method

Data / restraints / parameters

Goodness-of-fit on $F^{\wedge} 2$

Final $R$ indices [I>2sigma(I)]

$\mathrm{R}$ indices (all data)

Extinction coefficient

Largest diff. peak and hole yahong $8 t$

C36 H45 C12 N7 Ti

694.59

$173(2) \mathrm{K}$

0.71073 A

Triclinic, P-1

$\mathrm{a}=11.467(12)$ A alpha $=112.147(16)$ deg.

$\mathrm{b}=12.308(13)$ A beta $=95.438(17)$ deg.

$\mathrm{c}=15.270(16)$ A gamma $=111.167(17) \mathrm{deg}$.

$1794(3) \quad A \wedge 3$

$2,1.286 \mathrm{Mg} / \mathrm{m} \wedge 3$

$0.423 \mathrm{~mm}^{\wedge}-1$

732

$0.32 \times 0.23 \times 0.11 \mathrm{~mm}$

1.49 to $23.39 \mathrm{deg}$.

$-12<=h<=12, \quad-13<=\mathrm{k}<=13, \quad-17<=1<=16$

$15167 / 5182[\mathrm{R}($ int $)=0.0656]$

$98.9 \%$

Empirical

0.7565 and 0.5131

Full-matrix least-squares on $F^{\wedge} 2$

$5182 / 0 / 416$

1.057

$\mathrm{R} 1=0.0560, \mathrm{wR} 2=0.1365$

$\mathrm{R} 1=0.1057, \mathrm{wR} 2=0.1670$

$0.0014(10)$

0.861 and -0.747 e.A^-3

Table 2. Atomic coordinates ( $\mathrm{x}$ 10^4) and equivalent isotropic displacement parameters ( $\mathrm{A} \wedge 2 \times 10 \wedge 3)$ for $\mathrm{Ti}\left[\mathrm{NN}(\mathrm{H}) \mathrm{Ph}\right.$ ] [Bu ${ }^{\mathrm{t}}$-bpy] [dpma]. U(eq) is defined as one third of the trace of the orthogonalized Uij tensor. 


\begin{tabular}{|c|c|c|c|c|}
\hline $\mathrm{Ti}$ & $6939(1)$ & $5983(1)$ & $7365(1)$ & $26(1)$ \\
\hline $\mathrm{Cl}(1 \mathrm{~S})$ & $338(3)$ & $1622(4)$ & $7416(2)$ & $186(2)$ \\
\hline $\mathrm{Cl}(2 \mathrm{~S})$ & $-248(2)$ & $3410(2)$ & $8962(2)$ & $122(1)$ \\
\hline $\mathrm{N}(1)$ & $6925(3)$ & 7175 (3) & $8752(3)$ & 31 (1) \\
\hline $\mathrm{N}(2)$ & $4974(3)$ & $4880(3)$ & $6673(3)$ & $32(1)$ \\
\hline $\mathrm{N}(3)$ & $6199(4)$ & $4497(3)$ & $8067(3)$ & $32(1)$ \\
\hline $\mathrm{N}(4)$ & $8989(3)$ & 6409 (3) & $7980(3)$ & $28(1)$ \\
\hline$N(5)$ & $7428(3)$ & $4454(3)$ & $6322(3)$ & $26(1)$ \\
\hline$N(6)$ & $7192(3)$ & $6944(3)$ & $6767(3)$ & $30(1)$ \\
\hline$N(61)$ & $7237(4)$ & $7537(4)$ & $6178(3)$ & $39(1)$ \\
\hline$C(1 S)$ & $-238(7)$ & $1860(7)$ & $8391(6)$ & $91(2)$ \\
\hline C (11) & $7505(4)$ & $8508(4)$ & $9340(4)$ & $36(1)$ \\
\hline C (12) & $7569(5)$ & $8774(5)$ & $10298(4)$ & $46(1)$ \\
\hline C (13) & $6993(5)$ & $7565(5)$ & $10333(4)$ & $48(1)$ \\
\hline C (14) & $6619(4)$ & $6624(4)$ & $9380(3)$ & $34(1)$ \\
\hline C (21) & $4095(5)$ & $5127(5)$ & $6207(3)$ & $38(1)$ \\
\hline C (22) & $2856(5)$ & $4290(5)$ & $6094(4)$ & $47(1)$ \\
\hline C (23) & $2968(5)$ & $3470(5)$ & $6504(4)$ & $47(1)$ \\
\hline C (24) & $4260(4)$ & $3855(4)$ & $6848(3)$ & $34(1)$ \\
\hline C (31) & $5814(5)$ & $5191(4)$ & $8937(4)$ & $38(1)$ \\
\hline C (32) & $5020(5)$ & $3364(4)$ & $7314(4)$ & $39(1)$ \\
\hline C (33) & $7138(5)$ & $4064(5)$ & $8372(4)$ & $40(1)$ \\
\hline C (42) & $9532(4)$ & $5648(4)$ & $7464(3)$ & $24(1)$ \\
\hline$C(43)$ & $10804(4)$ & $5915(4)$ & $7804(3)$ & $29(1)$ \\
\hline C (44) & $11618(4)$ & $6988(4)$ & 8679 (3) & $27(1)$ \\
\hline$C(45)$ & $11031(4)$ & $7747(4)$ & $9191(3)$ & $35(1)$ \\
\hline$C(46)$ & $9753(4)$ & $7431(4)$ & $8831(3)$ & $35(1)$ \\
\hline C (52) & $8649(4)$ & $4543(4)$ & 6519 (3) & $25(1)$ \\
\hline C (53) & $9023(4)$ & $3680(4)$ & $5876(3)$ & $27(1)$ \\
\hline C (54) & $8164(4)$ & $2676(4)$ & $4982(3)$ & $26(1)$ \\
\hline C (55) & $6925(4)$ & $2611(4)$ & $4798(3)$ & $30(1)$ \\
\hline C (56) & $6597(4)$ & $3493(4)$ & $5470(3)$ & $28(1)$ \\
\hline$C(440)$ & $13031(4)$ & $7302(5)$ & $9030(3)$ & $38(1)$ \\
\hline C (441) & $13649(5)$ & $8378(5)$ & $10078(4)$ & $48(1)$ \\
\hline$C(442)$ & $13139(6)$ & $6099(6)$ & $9016(5)$ & $72(2)$ \\
\hline$C(443)$ & $13769(5)$ & $7734(7)$ & $8355(4)$ & $75(2)$ \\
\hline$C(540)$ & $8626(4)$ & $1767(4)$ & $4261(3)$ & $33(1)$ \\
\hline$C(541)$ & $9484(5)$ & $2537(5)$ & $3792(4)$ & $52(2)$ \\
\hline$C(542)$ & $9414(5)$ & $1316(5)$ & $4788(4)$ & $40(1)$ \\
\hline$C(543)$ & $7488(5)$ & $566(5)$ & $3442(4)$ & $52(2)$ \\
\hline$C(611)$ & $6816(4)$ & $8505(4)$ & $6336(3)$ & $31(1)$ \\
\hline C (612) & $6841(5)$ & $9030(5)$ & $5683(4)$ & $43(1)$ \\
\hline$C(613)$ & $6440(5)$ & $10000(5)$ & $5838(5)$ & $52(2)$ \\
\hline$C(614)$ & $6013(5)$ & 10455 (5) & $6660(5)$ & $52(2)$ \\
\hline$C(615)$ & $5975(5)$ & $9928(5)$ & $7307(4)$ & $45(1)$ \\
\hline$C(616)$ & $6362(4)$ & $8949(4)$ & $7158(3)$ & $34(1)$ \\
\hline
\end{tabular}

Table 3. Bond lengths [A] and angles [deg] for $\mathrm{Ti}[\mathrm{NN}(\mathrm{H}) \mathrm{Ph}]\left[\mathrm{Bu}^{\mathrm{t}}\right.$-bpy] [dpma] .

\begin{tabular}{ll}
\hline & \\
$\operatorname{Ti}-N(6)$ & $1.712(4)$ \\
$\operatorname{Ti}-N(2)$ & $2.073(4)$ \\
$\operatorname{Ti}-N(1)$ & $2.079(4)$ \\
$\operatorname{Ti}-\mathrm{N}(4)$ & $2.237(4)$ \\
$\operatorname{Ti}-\mathrm{N}(5)$ & $2.251(4)$ \\
$\mathrm{Ti}-\mathrm{N}(3)$ & $2.396(4)$ \\
$\mathrm{Cl}(1 \mathrm{~S})-\mathrm{C}(1 \mathrm{~S})$ & $1.654(7)$ \\
$\mathrm{Cl}(2 \mathrm{~S})-\mathrm{C}(1 \mathrm{~S})$ & $1.779(8)$ \\
$\mathrm{N}(1)-C(14)$ & $1.372(6)$ \\
$\mathrm{N}(1)-\mathrm{C}(11)$ & $1.389(6)$
\end{tabular}




\begin{tabular}{|c|c|}
\hline & \\
\hline$N(2)-C(21)$ & $1.365(6)$ \\
\hline$N(2)-C(24)$ & $1.372(6)$ \\
\hline$N(3)-C(33)$ & $1.472(6)$ \\
\hline$N(3)-C(31)$ & $1.488(6)$ \\
\hline$N(3)-C(32)$ & $1.493(6)$ \\
\hline$N(4)-C(46)$ & $1.339(6)$ \\
\hline$N(4)-C(42)$ & $1.359(5)$ \\
\hline$N(5)-C(56)$ & $1.338(5)$ \\
\hline$N(5)-C(52)$ & $1.359(5)$ \\
\hline$N(6)-N(61)$ & $1.350(5)$ \\
\hline $\mathrm{N}(61)-\mathrm{C}(611)$ & $1.390(6)$ \\
\hline$C(11)-C(12)$ & $1.361(7)$ \\
\hline$C(12)-C(13)$ & $1.418(7)$ \\
\hline$C(13)-C(14)$ & $1.377(7)$ \\
\hline$C(14)-C(31)$ & $1.492(7)$ \\
\hline$C(21)-C(22)$ & $1.368(7)$ \\
\hline$C(22)-C(23)$ & $1.408(7)$ \\
\hline$C(23)-C(24)$ & $1.362(7)$ \\
\hline$C(24)-C(32)$ & $1.489(7)$ \\
\hline$C(42)-C(43)$ & $1.373(6)$ \\
\hline$C(42)-C(52)$ & $1.485(6)$ \\
\hline$C(43)-C(44)$ & $1.394(6)$ \\
\hline$C(44)-C(45)$ & $1.393(6)$ \\
\hline$C(44)-C(440)$ & $1.514(6)$ \\
\hline$C(45)-C(46)$ & $1.370(6)$ \\
\hline$C(52)-C(53)$ & $1.378(6)$ \\
\hline$C(53)-C(54)$ & $1.398(6)$ \\
\hline$C(54)-C(55)$ & $1.388(6)$ \\
\hline$C(54)-C(540)$ & $1.535(6)$ \\
\hline$C(55)-C(56)$ & $1.379(6)$ \\
\hline$C(440)-C(443)$ & $1.522(7)$ \\
\hline$C(440)-C(442)$ & $1.522(7)$ \\
\hline$C(440)-C(441)$ & $1.525(7)$ \\
\hline$C(540)-C(542)$ & $1.527(6)$ \\
\hline$C(540)-C(541)$ & $1.533(7)$ \\
\hline$C(540)-C(543)$ & $1.535(7)$ \\
\hline$C(611)-C(612)$ & $1.374(6)$ \\
\hline$C(611)-C(616)$ & $1.400(6)$ \\
\hline$C(612)-C(613)$ & $1.377(7)$ \\
\hline$C(613)-C(614)$ & $1.383(8)$ \\
\hline$C(614)-C(615)$ & $1.367(7)$ \\
\hline$C(615)-C(616)$ & $1.377(6)$ \\
\hline$N(6)-T i-N(2)$ & $95.68(17)$ \\
\hline$N(6)-T i-N(1)$ & $103.82(18)$ \\
\hline$N(2)-T i-N(1)$ & $101.94(15)$ \\
\hline$N(6)-T i-N(4)$ & $100.13(16)$ \\
\hline $\mathrm{N}(2)-\mathrm{Ti}-\mathrm{N}(4)$ & $157.97(14)$ \\
\hline$N(1)-T i-N(4)$ & $89.13(14)$ \\
\hline$N(6)-T i-N(5)$ & $97.39(17)$ \\
\hline$N(2)-T i-N(5)$ & $91.60(15)$ \\
\hline$N(1)-T i-N(5)$ & $153.41(14)$ \\
\hline $\mathrm{N}(4)-\mathrm{Ti}-\mathrm{N}(5)$ & $71.30(14)$ \\
\hline$N(6)-T i-N(3)$ & $170.20(16)$ \\
\hline$N(2)-T i-N(3)$ & $74.60(15)$ \\
\hline$N(1)-T i-N(3)$ & $77.49(16)$ \\
\hline$N(4)-T i-N(3)$ & $89.58(13)$ \\
\hline$N(5)-T i-N(3)$ & $84.35(15)$ \\
\hline$C(14)-N(1)-C(11)$ & $105.5(4)$ \\
\hline $\mathrm{C}(14)-\mathrm{N}(1)-\mathrm{T} i$ & $117.2(3)$ \\
\hline $\mathrm{C}(11)-\mathrm{N}(1)-\mathrm{T} \mathrm{i}$ & $134.3(3)$ \\
\hline$C(21)-N(2)-C(24)$ & $106.0(4)$ \\
\hline $\mathrm{C}(21)-\mathrm{N}(2)-\mathrm{T} i$ & $131.3(3)$ \\
\hline $\mathrm{C}(24)-\mathrm{N}(2)-\mathrm{Ti}$ & $120.7(3)$ \\
\hline$C(33)-N(3)-C(31)$ & $109.4(4)$ \\
\hline$C(33)-N(3)-C(32)$ & $110.7(4)$ \\
\hline$C(31)-N(3)-C(32)$ & $109.4(4)$ \\
\hline $\mathrm{C}(33)-\mathrm{N}(3)-\mathrm{Ti}$ & $116.5(3)$ \\
\hline $\mathrm{C}(31)-\mathrm{N}(3)-\mathrm{Ti}$ & $105.1(3)$ \\
\hline$C(32)-N(3)-T i$ & $105.5(3)$ \\
\hline$C(46)-N(4)-C(42)$ & $116.8(4)$ \\
\hline $\mathrm{C}(46)-\mathrm{N}(4)-\mathrm{Ti}$ & $123.1(3)$ \\
\hline
\end{tabular}




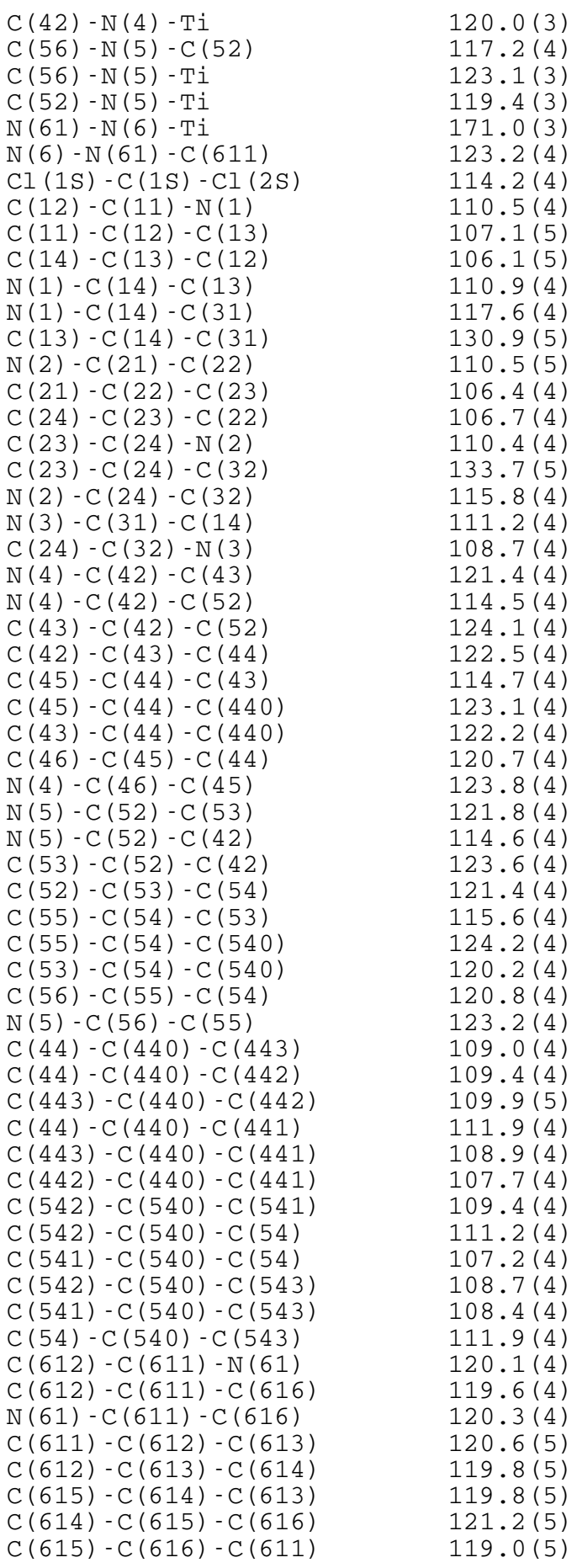

$117.2(4)$

123.1 (3)

$119.4(3)$

$171.0(3)$

$123.2(4)$

$114.2(4)$

$110.5(4)$

107.1 (5)

106.1 (5)

$110.9(4)$

$117.6(4)$

$130.9(5)$

$110.5(5)$

$106.4(4)$

106.7 (4)

$110.4(4)$

$133.7(5)$

$115.8(4)$

$111.2(4)$

108.7 (4)

$121.4(4)$

$114.5(4)$

$124.1(4)$

$122.5(4)$

$114.7(4)$

$123.1(4)$

122.2 (4)

$120.7(4)$

$123.8(4)$

$121.8(4)$

$114.6(4)$

$123.6(4)$

$121.4(4)$

$115.6(4)$

$124.2(4)$

$120.2(4)$

$120.8(4)$

$123.2(4)$

$109.0(4)$

$109.4(4)$

$109.9(5)$

$111.9(4)$

$108.9(4)$

$107.7(4)$

$109.4(4)$

$111.2(4)$

$107.2(4)$

$108.7(4)$

$108.4(4)$

$111.9(4)$

$120.1(4)$

$119.6(4)$

$120.3(4)$

$120.6(5)$

$119.8(5)$

$119.8(5)$

$121.2(5)$

$119.0(5)$ 
Symmetry transformations used to generate equivalent atoms:

Table 4. Anisotropic displacement parameters $(A \wedge 2 \times 10 \wedge 3)$ for $\mathrm{Ti}[\mathrm{NN}(\mathrm{H}) \mathrm{Ph}$ ] [Bu - bpy] [dpma] .

The anisotropic displacement factor exponent takes the form:

$-2 \mathrm{pi} \wedge 2[\mathrm{~h} \wedge 2 \mathrm{a} * \wedge 2 \mathrm{U} 11+\ldots+2 \mathrm{~h} \mathrm{k} a * \mathrm{~b} * \mathrm{U} 12]$

\begin{tabular}{|c|c|c|c|c|c|c|}
\hline & U11 & U2 2 & U33 & U2 3 & U13 & U12 \\
\hline $\mathrm{Ti}$ & $25(1)$ & $20(1)$ & $35(1)$ & $10(1)$ & $6(1)$ & $13(1)$ \\
\hline $\mathrm{Cl}(1 \mathrm{~S})$ & $152(3)$ & $297(4)$ & $76(2)$ & $52(2)$ & $47(2)$ & $90(3)$ \\
\hline $\mathrm{Cl}(2 \mathrm{~S})$ & $106(2)$ & $108(2)$ & $169(2)$ & $70(2)$ & $20(2)$ & $60(2)$ \\
\hline $\mathrm{N}(1)$ & $31(2)$ & $24(2)$ & $37(2)$ & $9(2)$ & $7(2)$ & $17(2)$ \\
\hline $\mathrm{N}(2)$ & $28(2)$ & $27(2)$ & $40(2)$ & $11(2)$ & $6(2)$ & $16(2)$ \\
\hline $\mathrm{N}(3)$ & $32(2)$ & $24(2)$ & $42(2)$ & $16(2)$ & $12(2)$ & $15(2)$ \\
\hline $\mathrm{N}(4)$ & $26(2)$ & $20(2)$ & $31(2)$ & $6(2)$ & $4(2)$ & $10(2)$ \\
\hline $\mathrm{N}(5)$ & $25(2)$ & $21(2)$ & $29(2)$ & $8(2)$ & $2(2)$ & $11(2)$ \\
\hline $\mathrm{N}(6)$ & $33(2)$ & $25(2)$ & $37(2)$ & $16(2)$ & $10(2)$ & $15(2)$ \\
\hline $\mathrm{N}(61)$ & $54(3)$ & $39(2)$ & $44(3)$ & $24(2)$ & $27(2)$ & $31(2)$ \\
\hline$C(1 S)$ & $76(5)$ & $109(6)$ & $101(6)$ & $57(5)$ & $38(4)$ & $37(5)$ \\
\hline $\mathrm{C}(11)$ & $37(3)$ & $28(3)$ & $44(3)$ & $11(2)$ & $8(2)$ & $19(2)$ \\
\hline$C(12)$ & $56(4)$ & $35(3)$ & $37(3)$ & $3(3)$ & $7(3)$ & $24(3)$ \\
\hline$C(13)$ & $56(4)$ & $52(4)$ & $34(3)$ & $16(3)$ & $9(3)$ & $26(3)$ \\
\hline$C(14)$ & $35(3)$ & $34(3)$ & $38(3)$ & $15(3)$ & $11(2)$ & $22(2)$ \\
\hline$C(21)$ & $36(3)$ & $39(3)$ & $35(3)$ & $7(2)$ & $3(2)$ & $25(3)$ \\
\hline$C(22)$ & $27(3)$ & $57(4)$ & $41(3)$ & $6(3)$ & $-1(2)$ & $20(3)$ \\
\hline$C(23)$ & $25(3)$ & $43(3)$ & $48(3)$ & $7(3)$ & $7(2)$ & $2(2)$ \\
\hline$C(24)$ & $27(3)$ & $30(3)$ & $34(3)$ & $8(2)$ & $6(2)$ & $9(2)$ \\
\hline$C(31)$ & $39(3)$ & $35(3)$ & $43(3)$ & $16(2)$ & $16(2)$ & $18(2)$ \\
\hline$C(32)$ & $38(3)$ & $26(3)$ & $50(3)$ & $17(3)$ & $16(3)$ & $10(2)$ \\
\hline$C(33)$ & $49(3)$ & $36(3)$ & $47(3)$ & $25(3)$ & $14(3)$ & $23(3)$ \\
\hline
\end{tabular}




\begin{tabular}{|c|c|c|c|c|c|c|}
\hline C (42) & $22(2)$ & $21(2)$ & $29(3)$ & $10(2)$ & $7(2)$ & $10(2)$ \\
\hline C (43) & $28(3)$ & $25(3)$ & $32(3)$ & $8(2)$ & $8(2)$ & $15(2)$ \\
\hline C (44) & $23(2)$ & $24(2)$ & $29(3)$ & $11(2)$ & $4(2)$ & $9(2)$ \\
\hline C (45) & $28(3)$ & $23(2)$ & $35(3)$ & $0(2)$ & $-2(2)$ & $8(2)$ \\
\hline C (46) & $33(3)$ & $25(3)$ & $40(3)$ & $5(2)$ & $9(2)$ & $16(2)$ \\
\hline$C(52)$ & $23(2)$ & $21(2)$ & $29(3)$ & $11(2)$ & $6(2)$ & $9(2)$ \\
\hline C (53) & $20(2)$ & $27(3)$ & 31 (3) & $10(2)$ & $5(2)$ & $10(2)$ \\
\hline C (54) & $29(3)$ & $19(2)$ & $28(3)$ & $11(2)$ & $7(2)$ & $9(2)$ \\
\hline C (55) & $30(3)$ & $22(2)$ & $27(3)$ & $4(2)$ & $0(2)$ & $9(2)$ \\
\hline C (56) & $21(2)$ & $28(3)$ & $34(3)$ & $13(2)$ & $3(2)$ & $10(2)$ \\
\hline$C(440)$ & $27(3)$ & $38(3)$ & $35(3)$ & $5(2)$ & $0(2)$ & $14(2)$ \\
\hline$C(441)$ & $34(3)$ & $49(3)$ & $41(3)$ & $4(3)$ & $-3(2)$ & $18(3)$ \\
\hline$C(442)$ & $54(4)$ & $59(4)$ & $76(4)$ & $1(3)$ & $-22(3)$ & $37(3)$ \\
\hline$C(443)$ & $27(3)$ & $107(5)$ & $53(4)$ & $23(4)$ & $10(3)$ & $3(3)$ \\
\hline$C(540)$ & $32(3)$ & $31(3)$ & $30(3)$ & $6(2)$ & $8(2)$ & $15(2)$ \\
\hline$C(541)$ & $59(4)$ & $61(4)$ & $47(3)$ & $28(3)$ & $27(3)$ & $33(3)$ \\
\hline$C(542)$ & $43(3)$ & $31(3)$ & $42(3)$ & $10(2)$ & $9(2)$ & $19(2)$ \\
\hline$C(543)$ & $47(3)$ & $48(3)$ & $39(3)$ & $-5(3)$ & $5(3)$ & $26(3)$ \\
\hline$C(611)$ & $32(3)$ & $22(2)$ & $38(3)$ & $11(2)$ & $9(2)$ & $11(2)$ \\
\hline$C(612)$ & $53(3)$ & $43(3)$ & $53(3)$ & $33(3)$ & $24(3)$ & $26(3)$ \\
\hline$C(613)$ & $54(4)$ & $40(3)$ & $76(4)$ & $41(3)$ & $20(3)$ & $19(3)$ \\
\hline$C(614)$ & $50(4)$ & $25(3)$ & $73(4)$ & $16(3)$ & $-1(3)$ & $18(3)$ \\
\hline$C(615)$ & $48(3)$ & $34(3)$ & $37(3)$ & $-4(3)$ & $-1(3)$ & $24(3)$ \\
\hline$C(616)$ & $39(3)$ & $31(3)$ & $29(3)$ & $9(2)$ & $4(2)$ & $16(2)$ \\
\hline
\end{tabular}

Table 5. Hydrogen coordinates $(\mathrm{x} 10 \wedge 4)$ and isotropic displacement parameters $(\mathrm{A} \wedge 2 \times 10 \wedge 3)$ for $\mathrm{Ti}\left[\mathrm{NN}(\mathrm{H}) \mathrm{Ph}\right.$ ] [Bu ${ }^{\mathrm{t}}$-bpy] [dpma] .

\begin{tabular}{|c|c|c|c|c|}
\hline & $\mathrm{x}$ & $\mathrm{y}$ & z & $\mathrm{U}(\mathrm{eq})$ \\
\hline $\mathrm{H}(61 \mathrm{~A})$ & 7542 & 7304 & 5680 & 47 \\
\hline $\mathrm{H}(1 \mathrm{SA})$ & 280 & 1757 & 8868 & 110 \\
\hline $\mathrm{H}(1 \mathrm{SB})$ & -1118 & 1194 & 8197 & 110 \\
\hline $\mathrm{H}(11 \mathrm{~A})$ & 7807 & 9133 & 9112 & 44 \\
\hline $\mathrm{H}(12 \mathrm{~A})$ & 7923 & 9593 & 10829 & 55 \\
\hline$H(13 A)$ & 6890 & 7434 & 10887 & 57 \\
\hline $\mathrm{H}(21 \mathrm{~A})$ & 4308 & 5774 & 5996 & 45 \\
\hline $\mathrm{H}(22 \mathrm{~A})$ & 2092 & 4267 & 5803 & 57 \\
\hline $\mathrm{H}(23 \mathrm{~A})$ & 2290 & 2795 & 6534 & 57 \\
\hline $\mathrm{H}(31 \mathrm{~A})$ & 5915 & 4880 & 9423 & 45 \\
\hline $\mathrm{H}(31 \mathrm{~B})$ & 4907 & 5004 & 8741 & 45 \\
\hline $\mathrm{H}(32 \mathrm{~A})$ & 5273 & 2789 & 6820 & 47 \\
\hline $\mathrm{H}(32 \mathrm{~B})$ & 4495 & 2880 & 7620 & 47 \\
\hline $\mathrm{H}(33 \mathrm{~A})$ & 6742 & 3475 & 8640 & 60 \\
\hline $\mathrm{H}(33 \mathrm{~B})$ & 7884 & 4804 & 8860 & 60 \\
\hline $\mathrm{H}(33 \mathrm{C})$ & 7400 & 3633 & 7814 & 60 \\
\hline $\mathrm{H}(43 \mathrm{~A})$ & 11135 & 5359 & 7436 & 35 \\
\hline $\mathrm{H}(45 \mathrm{~A})$ & 11512 & 8475 & 9784 & 41 \\
\hline $\mathrm{H}(46 \mathrm{~A})$ & 9395 & 7958 & 9198 & 42 \\
\hline $\mathrm{H}(53 \mathrm{~A})$ & 9864 & 3766 & 6040 & 33 \\
\hline $\mathrm{H}(55 \mathrm{~A})$ & 6310 & 1965 & 4215 & 36 \\
\hline $\mathrm{H}(56 \mathrm{~A})$ & 5757 & 3417 & 5323 & 34 \\
\hline $\mathrm{H}(44 \mathrm{D})$ & 13592 & 9151 & 10109 & 72 \\
\hline $\mathrm{H}(44 \mathrm{E})$ & 14544 & 8554 & 10273 & 72 \\
\hline $\mathrm{H}(44 \mathrm{~F})$ & 13199 & 8108 & 10511 & 72 \\
\hline $\mathrm{H}(44 \mathrm{~A})$ & 12747 & 5400 & 8365 & 108 \\
\hline $\mathrm{H}(44 \mathrm{~B})$ & 12700 & 5852 & 9463 & 108 \\
\hline $\mathrm{H}(44 \mathrm{C})$ & 14037 & 6286 & 9211 & 108 \\
\hline $\mathrm{H}(44 \mathrm{G})$ & 13386 & 7061 & 7693 & 112 \\
\hline $\mathrm{H}(44 \mathrm{H})$ & 14659 & 7900 & 8558 & 112 \\
\hline $\mathrm{H}(44 \mathrm{I})$ & 13726 & 8512 & 8388 & 112 \\
\hline $\mathrm{H}(54 \mathrm{D})$ & 8986 & 2809 & 3452 & 78 \\
\hline $\mathrm{H}(54 \mathrm{E})$ & 9795 & 1998 & 3337 & 78 \\
\hline $\mathrm{H}(54 \mathrm{~F})$ & 10208 & 3287 & 4295 & 78 \\
\hline
\end{tabular}




\begin{tabular}{lrrrl}
$\mathrm{H}(54 \mathrm{~A})$ & 8877 & 831 & 5080 & 60 \\
$\mathrm{H}(54 \mathrm{~B})$ & 10143 & 2059 & 5291 & 60 \\
$\mathrm{H}(54 \mathrm{C})$ & 9716 & 775 & 4326 & 60 \\
$\mathrm{H}(54 \mathrm{G})$ & 6942 & 69 & 3722 & 78 \\
$\mathrm{H}(54 \mathrm{H})$ & 7816 & 41 & 2992 & 78 \\
$\mathrm{H}(54 \mathrm{I})$ & 6993 & 838 & 3098 & 78 \\
$\mathrm{H}(61 \mathrm{~B})$ & 7131 & 8727 & 5132 & 52 \\
$\mathrm{H}(61 \mathrm{~F})$ & 6457 & 10348 & 5392 & 63 \\
$\mathrm{H}(61 \mathrm{D})$ & 5752 & 11117 & 6772 & 63 \\
$\mathrm{H}(61 \mathrm{E})$ & 5684 & 10236 & 7856 & 54 \\
$\mathrm{H}(61 \mathrm{C})$ & 6321 & 8589 & 7598 & 41 \\
\hline
\end{tabular}

\section{B. Discussion on modeling the electron transfer, Gaussian Fits, and fitting parameters for complexes 5-8}

According to dielectric continuum theory and the Marcus-Hush model, the electron transfer can be modeled as nonpenetrating spheres encompassing the electron donor and acceptor. ${ }^{1}$ The energy of the optical transition given by Equation 1,

$$
E_{o p}=\lambda_{i}+\lambda_{o}+\Delta E^{\circ}=\lambda_{i}+e^{2}\left(\frac{1}{2 a_{1}}+\frac{1}{2 a_{2}}-\frac{1}{d}\right)\left(\frac{1}{D_{o p}}-\frac{1}{D_{s}}\right)+\Delta \mathrm{E}^{\circ}
$$

where $\lambda_{\mathrm{i}}$ and $\lambda_{\mathrm{o}}$ are contributions from internal and external reorganization, $e$ is the unit electron charge, $D_{o p}$ and $D_{s}$ are the optical and static dielectric constants of the medium $\left(D_{\mathrm{op}}=\mathrm{n}^{2}\right.$, where $\mathrm{n}$ is the index of refraction of the medium), $a_{1}$ and $a_{2}$ are the molecular radii of the donor and acceptor spheres, $d$ is the separation between the two spheres, and $\Delta \mathrm{E}^{\circ}$ being the internal energy difference between the two states involved in the transition. The solvent dependence can be verified using eq 1 to obtain a linear fit with $\mathrm{E}_{\mathrm{op}}-\Delta \mathrm{E}^{\circ}$ versus $1 / D_{\mathrm{op}}-1 / D_{\mathrm{s}}$.

1. (a) Goldsby, K. A.; Meyer, T. J. Inorg. Chem. 1984, 23, 3002. (b) Chen, P.; Meyer, T. J. Chem. Rev. 1998, 98, 1439. (c) Blackbourn, R. L.; Hupp, J. T. J. Phys. Chem. 1990, 94, 1788. (d) Hupp, J. T.; Dong, Y.; Blackbourn, R. L.; Lu, H. J. Phys. Chem. 1993, 97, 3278. 


$$
\Delta v_{1 / 2}=\left[16(\ln 2) k_{\mathrm{B}} \mathrm{T}\left(\mathrm{E}_{\mathrm{op}}-\Delta \mathrm{E}^{\circ}\right)\right]^{1 / 2}
$$

The low energy peak for complexes 5-8 were isolated and fit to one Gaussian. The internal energy difference between the two states involved in the transition $\left(\Delta \mathrm{E}^{\circ}\right)$, is calculated using the peak width from the Hush equations as shown in Equation 2. A representative plot of $E_{o p}-\Delta E^{\circ}$ versus $1 / D_{o p}-1 / D_{s}$ is shown in SI Figure 1 for complex 5. Gaussian fits did not give reliable results for complexes 1-4. Due to mixing with the high energy peaks, isolation of the low energy peak was not accurate leading to poor Gaussian fits in these compounds. Considering the complexes that were easily modeled did not submit to accurate analysis, no further attempts to analyze the dimethylhydrazido(2-) complexes were made.

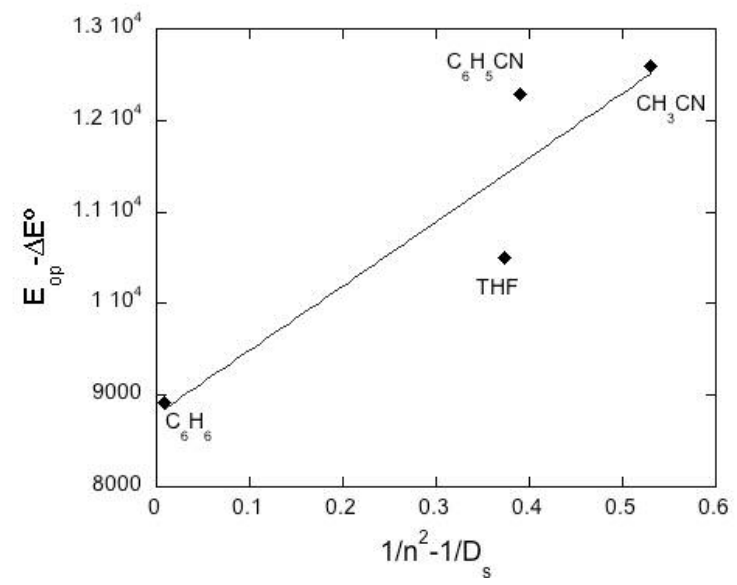

SI Figure 1. Plot of $E_{o p}-\Delta E^{\circ}$ versus $1 / D_{o p}-1 / D_{s}$ for 5

Extrapolation of $E_{o p}-\Delta E^{\circ}$ versus $1 / D_{o p}-1 / D_{s}$ to the energy axis (y-axis) gives $\lambda_{i}=7000$ $10000 \mathrm{~cm}^{-1}$ (SI Table 1). The high errors associated with the derived values might be an artifact of the two-sphere model as applied to this particular system. The model holds for two non-penetrating spheres, which may not be the case for these complexes.

Table 1. Linear fit data of $\mathrm{E}_{\mathrm{op}}-\Delta \mathrm{E}^{\circ}$ versus $1 / \mathrm{D}_{\mathrm{op}}-1 / \mathrm{D}_{\mathrm{s}}$ for complexes 5-8. 


\begin{tabular}{|c|c|c|c|}
\hline Complex & Slope & $\begin{array}{c}\text { Intercept } \\
\left(\mathrm{cm}^{-1}\right)\end{array}$ & $\mathrm{R}$ \\
\hline $\mathbf{5}$ & $7009[2181]$ & $8796[825]$ & 0.915 \\
\hline $\mathbf{6}$ & $7630[2820]$ & $7630[1067]$ & 0.886 \\
\hline $\mathbf{7}$ & $8564[3941]$ & $8394[1491]$ & 0.838 \\
\hline $\mathbf{8}$ & $8167[2876]$ & $10298[1088]$ & 0.895 \\
\hline
\end{tabular}

All the gaussian fits were done using OriginPro 7.5.

The equation used to make the plot was

$\mathrm{y}=\mathrm{y}_{0}+\left(\mathrm{A} /\left(\mathrm{w}^{*}(\pi / 2)^{1 / 2}\right)\right) * \exp \left(-2 *\left(\left(\mathrm{x}-\mathrm{x}_{\mathrm{c}}\right) / \mathrm{w}\right)^{2}\right)$

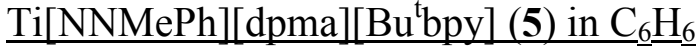

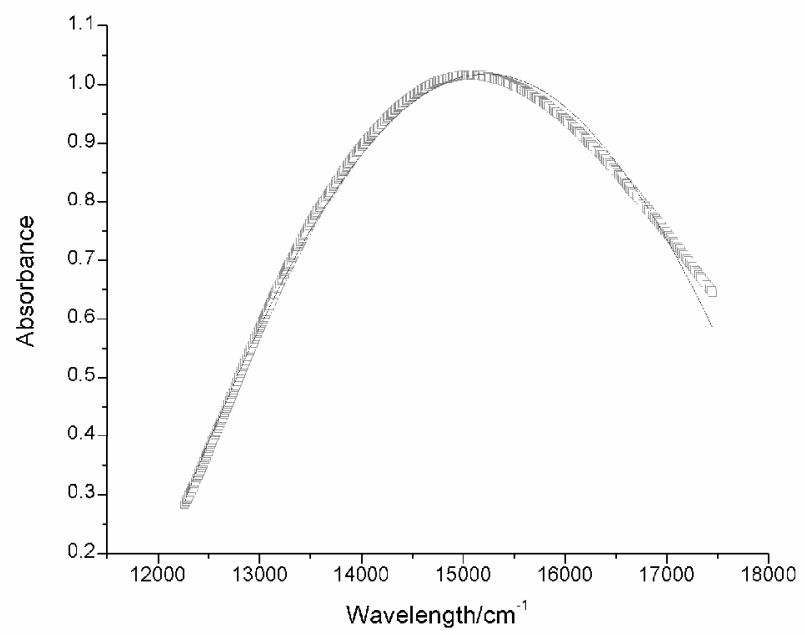

Data: $\operatorname{Ti}[\mathrm{NNMePh}][\mathrm{dpma}]\left[\mathrm{Bu}^{\mathrm{t}}\right.$ bpy $]: \mathrm{C}_{6} \mathrm{H}_{6}$

Model: Gauss

Equation: $\mathrm{y}=\mathrm{y}_{0}+\left(\mathrm{A} /\left(\mathrm{w}^{*}(\pi / 2)^{1 / 2}\right)\right) * \exp \left(-2 *\left(\left(\mathrm{x}-\mathrm{x}_{\mathrm{c}}\right) / \mathrm{w}\right)^{2}\right)$

Weighting:

y No weighting

$\mathrm{Chi}^{\wedge}$ 2/DoF $=0.00018$

$\mathrm{R}^{\wedge} 2=0.99607$ 


\begin{tabular}{lll} 
& \multicolumn{2}{c}{ Error } \\
y0 & -22.18661 & 15.44594 \\
xc & 15224.83963 & 2.99664 \\
w & 23197.95014 & 7823.12607 \\
A & 674509.45194 & 676526.43637
\end{tabular}




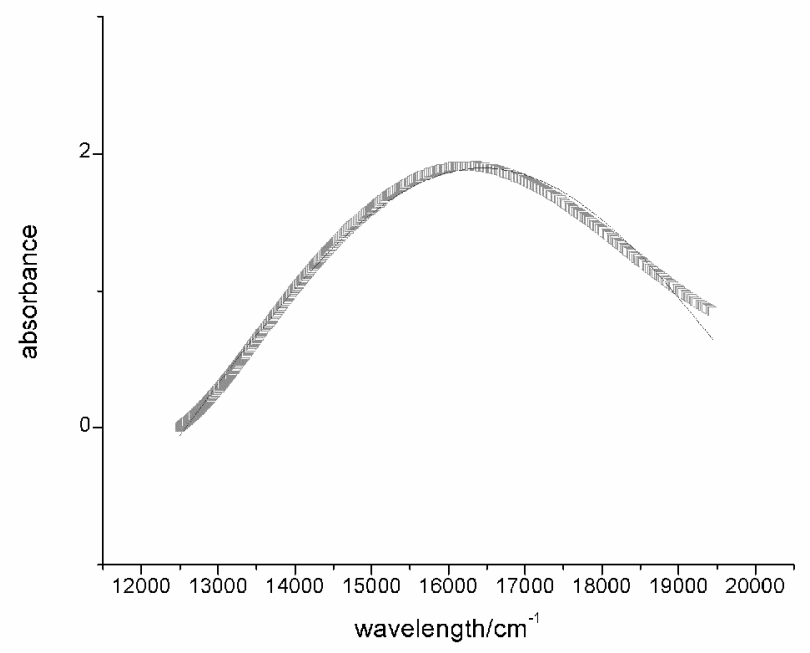

Data:Ti[NN(Me)Ph][Bu $\left.{ }^{t} b p y\right][d p m a]: T H F$

Model: Gauss

Equation: $\mathrm{y}=\mathrm{y}_{0}+\left(\mathrm{A} /\left(\mathrm{w}^{*}(\pi / 2)^{1 / 2}\right)\right) * \exp \left(-2 *\left(\left(\mathrm{x}-\mathrm{x}_{\mathrm{c}}\right) / \mathrm{w}\right)^{2}\right)$

Weighting:

y No weighting

$\mathrm{Chi}^{\wedge}{ }^{\wedge} 2 / \mathrm{DoF} \quad=0.00237$

$\mathrm{R}^{\wedge} 2=0.99312$

$\begin{array}{lll} & & \text { Error } \\ \text { y0 } & -2.76618 & \text { 0.19224 } \\ \text { xc } & 16449.76191 & 4.84561 \\ \text { W } & 7577.51792 & 193.11128 \\ \text { A } & 44243.2882 & 2930.01569\end{array}$




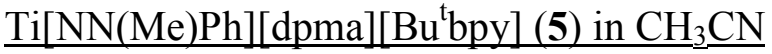

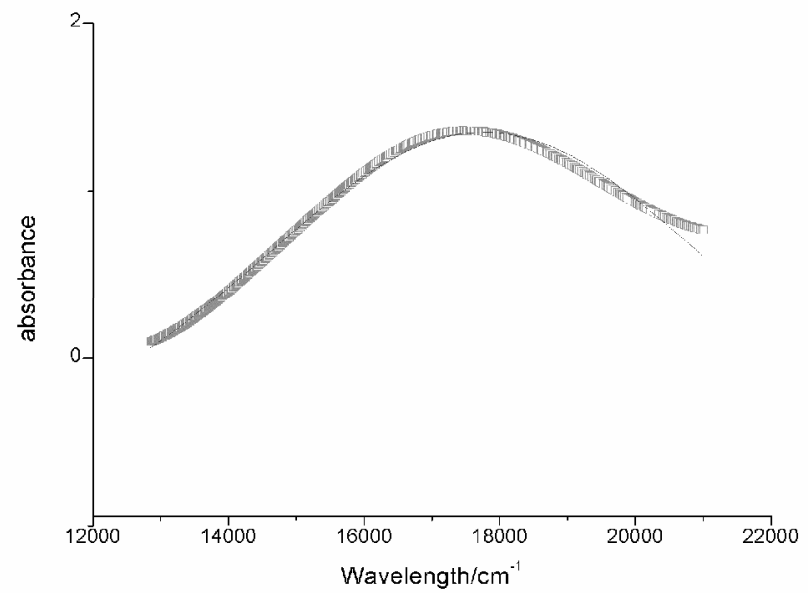

Data: $\operatorname{Ti}[\mathrm{NN}(\mathrm{Me}) \mathrm{Ph}]\left[\mathrm{Bu}^{\mathrm{t}}\right.$ bpy][dpma]: Acetonitrile Model: Gauss

Equation: $\mathrm{y}=\mathrm{y}_{0}+\left(\mathrm{A} /\left(\mathrm{w}^{*}(\pi / 2)^{1 / 2}\right)\right) * \exp \left(-2 *\left(\left(\mathrm{x}-\mathrm{x}_{\mathrm{c}}\right) / \mathrm{w}\right)^{2}\right)$

Weighting:

y No weighting

$\mathrm{Chi}^{\wedge}$ 2/DoF $=0.00107$

$\mathrm{R}^{\wedge} 2=0.99361$

\begin{tabular}{lll} 
& \multicolumn{2}{c}{ Error } \\
y0 & -0.52877 & 0.0286 \\
xc & 17775.30254 & 6.25607 \\
w & 6466.66341 & 76.76371 \\
A & 15226.36684 & 399.25366
\end{tabular}




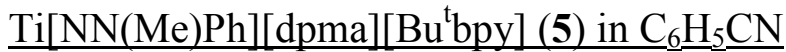

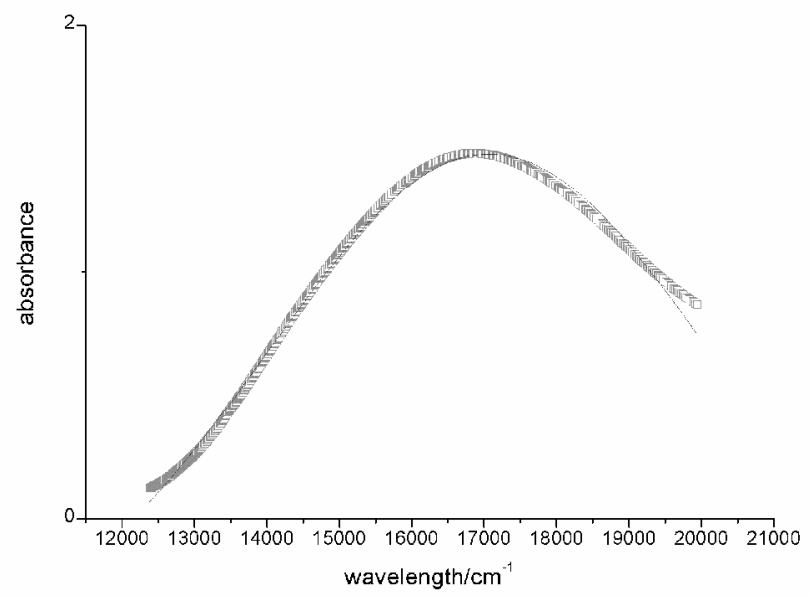

Data: $\operatorname{Ti}[\mathrm{NN}(\mathrm{Me}) \mathrm{Ph}]\left[\mathrm{Bu}^{\mathrm{t}} \mathrm{bpy}\right][\mathrm{dpma}]$ :benzonitrile

Model: Gauss

Equation: $\mathrm{y}=\mathrm{y}_{0}+\left(\mathrm{A} /\left(\mathrm{w} *(\pi / 2)^{1 / 2}\right)\right)^{*} \exp \left(-2 *\left(\left(\mathrm{x}-\mathrm{x}_{\mathrm{c}}\right) / \mathrm{w}\right)^{2}\right)$

Weighting:

y No weighting

$\mathrm{Chi}^{\wedge} 2 / \mathrm{DoF}=0.00074$

$\mathrm{R}^{\wedge} 2=0.99637$

\begin{tabular}{lll} 
& & \multicolumn{2}{c}{ Error } \\
y0 & -0.56279 & 0.02287 \\
xc & 17067.39877 & 4.69153 \\
w & 6108.17573 & 54.13825 \\
A & 15621.12557 & 303.0848
\end{tabular}




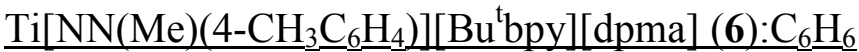

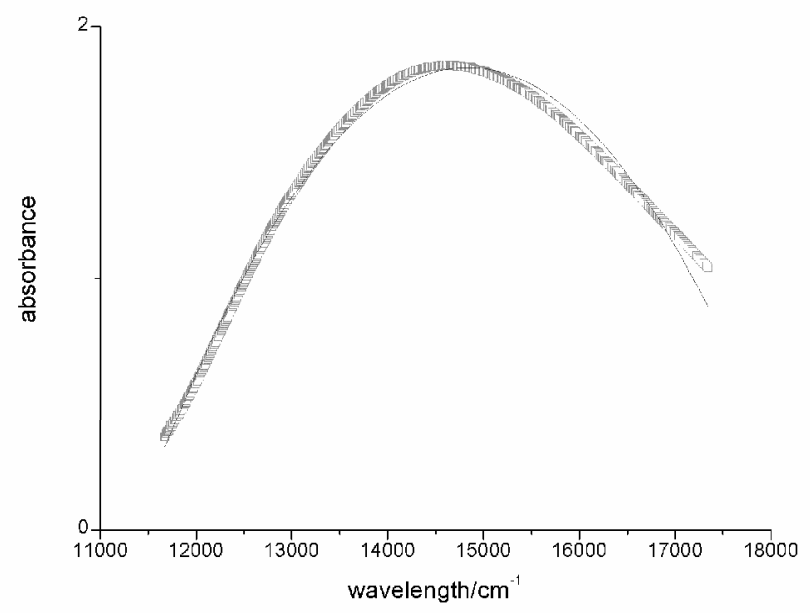

Data: Ti[NN(Me)(4- $\left.\left.\mathrm{CH}_{3} \mathrm{C}_{6} \mathrm{H}_{4}\right)\right]\left[\mathrm{Bu}^{\mathrm{t} b p y}\right][\mathrm{dpma}]: \mathrm{C}_{6} \mathrm{H}_{6}$ Model: Gauss

Equation: $\mathrm{y}=\mathrm{y}_{0}+\left(\mathrm{A} /\left(\mathrm{w} *(\pi / 2)^{1 / 2}\right)\right)^{*} \exp \left(-2 *\left(\left(\mathrm{x}-\mathrm{x}_{\mathrm{c}}\right) / \mathrm{w}\right)^{2}\right)$

Weighting:

y No weighting

$\mathrm{Chi}^{\wedge} 2 / \mathrm{DoF}=0.00152$

$\mathrm{R}^{\wedge} 2=0.99192$

\begin{tabular}{lll} 
& & \multicolumn{2}{c}{ Error } \\
y0 & -40.38619 & 33.77575 \\
xc & 14842.45188 & 4.09938 \\
w & 23436.79238 & 9511.14239 \\
A & 1240276.1452 & 1495389.57312
\end{tabular}




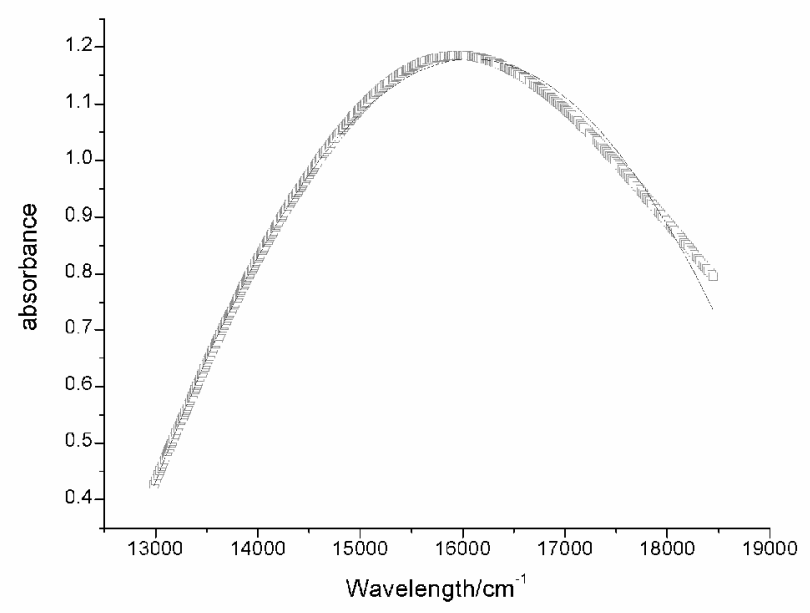

Data: Ti[NN(Me) $\left.\left(4-\mathrm{CH}_{3} \mathrm{C}_{6} \mathrm{H}_{4}\right)\right]\left[\mathrm{Bu}^{\mathrm{t}}\right.$ bpy] $[\mathrm{dpma}]$ :THF Model: Gauss

Equation: $\mathrm{y}=\mathrm{y}_{0}+\left(\mathrm{A} /\left(\mathrm{w}^{*}(\pi / 2)^{1 / 2}\right)\right) * \exp \left(-2 *\left(\left(\mathrm{x}-\mathrm{x}_{\mathrm{c}}\right) / \mathrm{w}\right)^{2}\right)$

Weighting:

y No weighting

$\mathrm{Chi}^{\wedge} 2 / \mathrm{DoF} \quad=0.00018$

$\mathrm{R}^{\wedge} 2=0.99616$

\begin{tabular}{lll} 
& & \multicolumn{2}{c}{ Error } \\
y0 & -9.09168 & 2.93154 \\
xc & 16093.3231 & 3.15642 \\
w & 15890.25675 & 2337.53045 \\
A & 204530.81321 & 88455.61753
\end{tabular}




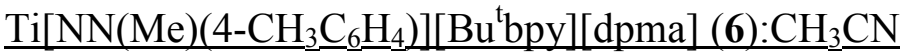

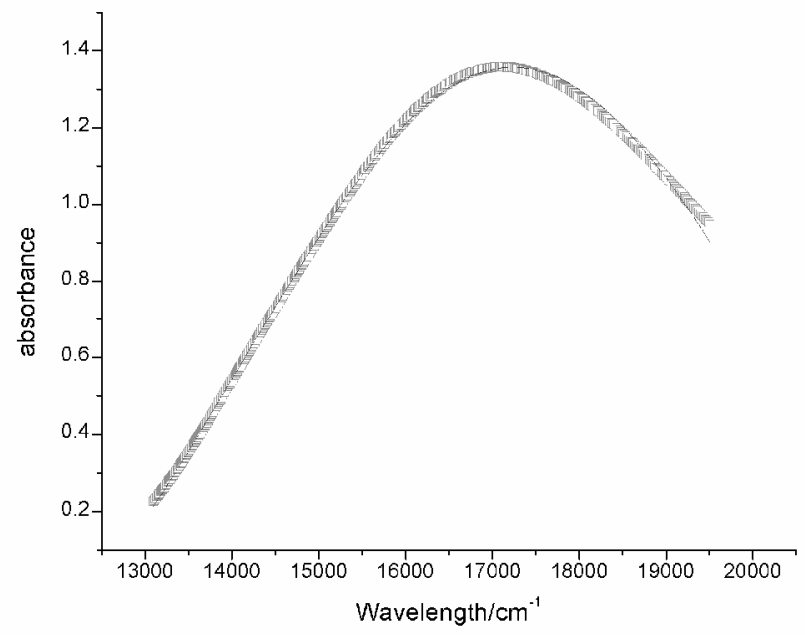

Data: Ti[NN(Me)(4- $\left.\left.\mathrm{CH}_{3} \mathrm{C}_{6} \mathrm{H}_{4}\right)\right]\left[\mathrm{Bu}^{\mathrm{t} b p y}\right][\mathrm{dpma}]$ :acetonitrile Model: Gauss

Equation: $\mathrm{y}=\mathrm{y}_{0}+\left(\mathrm{A} /\left(\mathrm{w} *(\pi / 2)^{1 / 2}\right)\right)^{*} \exp \left(-2 *\left(\left(\mathrm{x}-\mathrm{x}_{\mathrm{c}}\right) / \mathrm{w}\right)^{2}\right)$

Weighting:

y No weighting

$\mathrm{Chi}^{\wedge} 2 / \mathrm{DoF} \quad=0.00011$

$\mathrm{R}^{\wedge} 2=0.99911$

\begin{tabular}{lll} 
& & \multicolumn{2}{c}{ Error } \\
y0 & -0.58847 & 0.01731 \\
xc & 17236.64999 & 2.56136 \\
W & 6215.36192 & 39.82996 \\
A & 15154.33627 & 227.23529
\end{tabular}




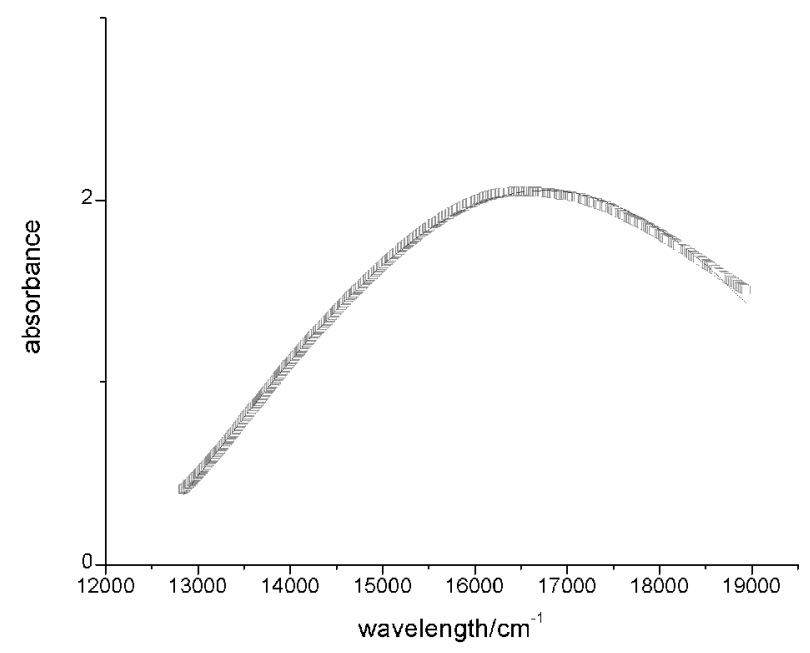

Data: Ti[NN(Me) $\left.\left(4-\mathrm{CH}_{3} \mathrm{C}_{6} \mathrm{H}_{4}\right)\right][\mathrm{Bu}$ tbpy][dpma]:benzonitrile Model: Gauss

Equation: $\mathrm{y}=\mathrm{y}_{0}+\left(\mathrm{A} /\left(\mathrm{w}^{*}(\pi / 2)^{1 / 2}\right)\right) * \exp \left(-2 *\left(\left(\mathrm{x}-\mathrm{x}_{\mathrm{c}}\right) / \mathrm{w}\right)^{2}\right)$ Weighting:

y No weighting

$\mathrm{Chi}^{\wedge} 2 / \mathrm{DoF} \quad=0.00031$

$\mathrm{R}^{\wedge} 2=0.99883$

$\begin{array}{lll} & & \text { Error } \\ \text { y0 } & -1.98216 & 0.07613 \\ \text { xc } & 16754.70174 & 2.90608 \\ \text { w } & 7559.34402 & 89.40894 \\ \text { A } & 38218.60329 & 1163.86477\end{array}$




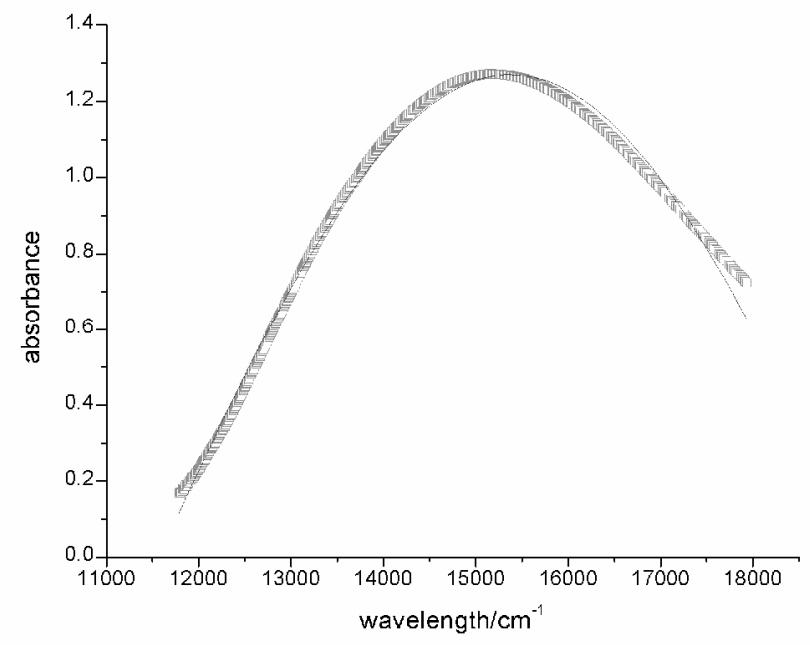

Data: $\mathrm{Ti}\left[\mathrm{NN}(\mathrm{Me})\left(4-\mathrm{FC}_{6} \mathrm{H}_{4}\right)\right]\left[\mathrm{Bu}^{\mathrm{t}} \mathrm{bpy}\right][\mathrm{dpma}]$ :benzonitrile Model: Gauss

Equation: $\mathrm{y}=\mathrm{y}_{0}+\left(\mathrm{A} /\left(\mathrm{w} *(\pi / 2)^{1 / 2}\right)\right) * \exp \left(-2 *\left(\left(\mathrm{x}-\mathrm{x}_{\mathrm{c}}\right) / \mathrm{w}\right)^{2}\right)$ Weighting:

y No weighting

$\mathrm{Chi}^{\wedge} 2 / \mathrm{DoF} \quad=0.00065$

$\mathrm{R}^{\wedge} 2=0.99446$

$\begin{array}{lll} & & \text { Error } \\ \text { y0 } & -2.30368 & 0.19054 \\ \text { xc } & 15380.45228 & 4.1917 \\ \text { w } & 8124.04293 & 252.72058 \\ \text { A } & 36383.68124 & 3058.87816\end{array}$




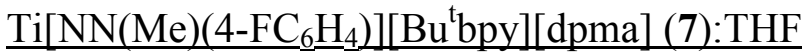

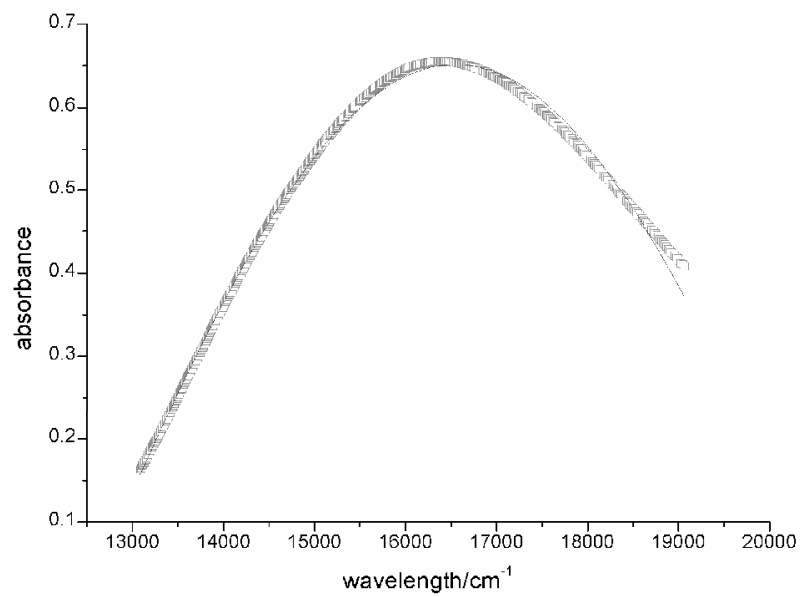

Data: Ti[NN(Me) $\left.\left(4-\mathrm{FC}_{6} \mathrm{H}_{4}\right)\right]\left[\mathrm{Bu}^{\mathrm{t} b p y}\right][\mathrm{dpma}]: \mathrm{THF}$ Model: Gauss

Equation: $\mathrm{y}=\mathrm{y}_{0}+\left(\mathrm{A} /\left(\mathrm{w}^{*}(\pi / 2)^{1 / 2}\right)\right) * \exp \left(-2 *\left(\left(\mathrm{x}-\mathrm{x}_{\mathrm{c}}\right) / \mathrm{w}\right)^{2}\right)$ Weighting:

y No weighting

$\mathrm{Chi}^{\wedge} 2 / \mathrm{DoF} \quad=0.00007$

$\mathrm{R}^{\wedge} 2=0.99685$

\begin{tabular}{lll} 
& & \multicolumn{2}{c}{ Error } \\
y0 & -1.19679 & 0.10502 \\
xc & 16552.3305 & 3.25399 \\
w & 8768.10819 & 281.59871 \\
A & 20312.48254 & 1801.58052
\end{tabular}




\section{$\left.\left.\underline{\operatorname{Ti}\left[\mathrm{NN}(\mathrm{Me})\left(4-\mathrm{FC}_{6}\right.\right.} \underline{\mathrm{H}}_{4}\right)\right]\left[\mathrm{Bu}{ }^{\mathrm{t} b p y}\right][\mathrm{dpma}](7): \mathrm{CH}_{3} \underline{\mathrm{CN}}$}

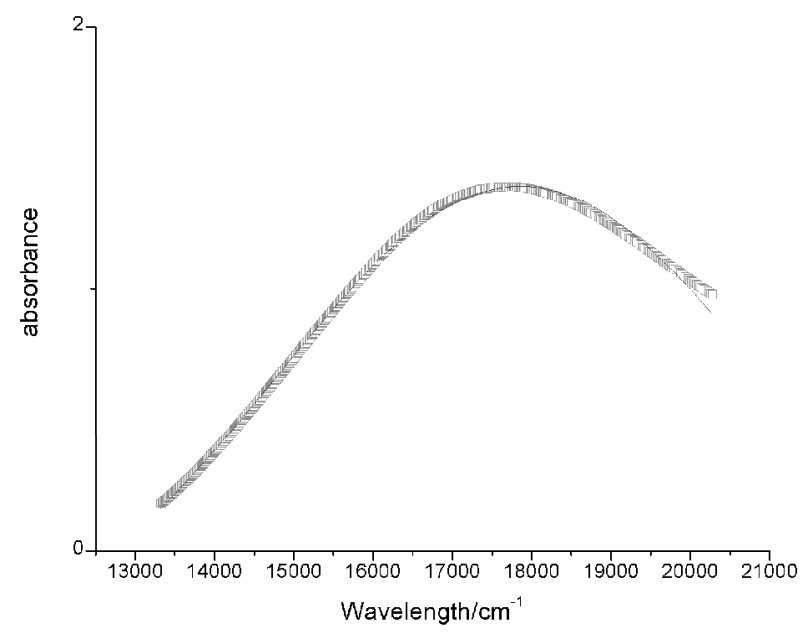

Data: $\mathrm{Ti}\left[\mathrm{NN}(\mathrm{Me})\left(4-\mathrm{FC}_{6} \mathrm{H}_{4}\right)\right]\left[\mathrm{Bu}^{\mathrm{t}}\right.$ bpy $][$ dpma]:acetonitrile Model: Gauss

Equation: $\mathrm{y}=\mathrm{y}_{0}+\left(\mathrm{A} /\left(\mathrm{w} *(\pi / 2)^{1 / 2}\right)\right)^{*} \exp \left(-2 *\left(\left(\mathrm{x}-\mathrm{x}_{\mathrm{c}}\right) / \mathrm{w}\right)^{2}\right)$ Weighting:

y No weighting

$\mathrm{Chi}^{\wedge} 2 / \mathrm{DoF} \quad=0.00028$

$\mathrm{R}^{\wedge} 2=0.99822$

\begin{tabular}{lll} 
& & \multicolumn{1}{c}{ Error } \\
y0 & -0.50479 & 0.01922 \\
xc & 17863.64803 & 3.99107 \\
w & 6270.98713 & 48.91729 \\
A & 14902.3192 & 259.94253
\end{tabular}




\section{$\left.\left.\underline{\operatorname{Ti}\left[N N(\mathrm{Me})\left(4-\mathrm{FC}_{6}\right.\right.} \underline{\mathrm{H}}_{4}\right)\right]\left[\mathrm{Bu}{ }^{\mathrm{t} b p y}\right][\mathrm{dpma}](7): \mathrm{C}_{6} \underline{\mathrm{H}_{5}} \underline{\mathrm{CN}}$}

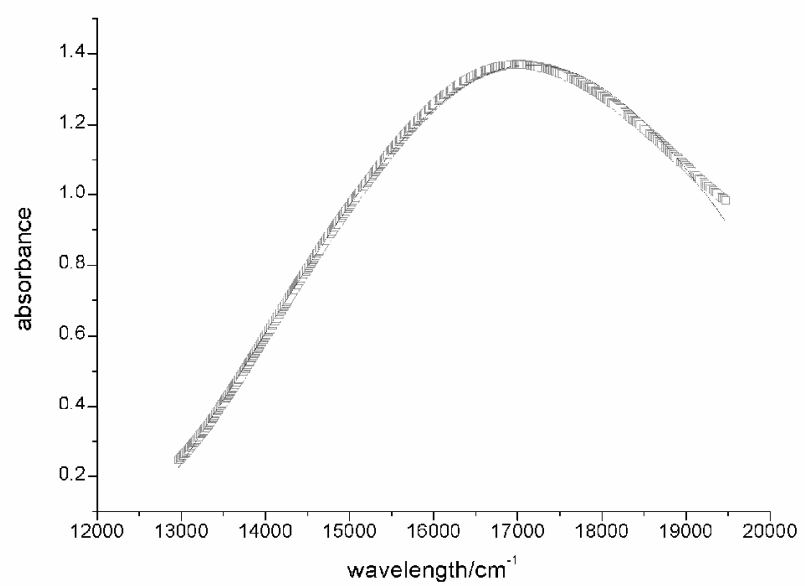

Data: $\mathrm{Ti}\left[\mathrm{NN}(\mathrm{Me})\left(4-\mathrm{FC}_{6} \mathrm{H}_{4}\right)\right][$ Butbpy][dpma]:benzonitrile Model: Gauss

Equation: $\mathrm{y}=\mathrm{y}_{0}+\left(\mathrm{A} /\left(\mathrm{w} *(\pi / 2)^{1 / 2}\right)\right) * \exp \left(-2 *\left(\left(\mathrm{x}-\mathrm{x}_{\mathrm{c}}\right) / \mathrm{w}\right)^{2}\right)$

Weighting:

y No weighting

$\mathrm{Chi}^{\wedge} 2 / \mathrm{DoF} \quad=0.00017$

$\mathrm{R}^{\wedge} 2=0.9987$

\begin{tabular}{lll} 
& \multicolumn{2}{c}{ Error } \\
y0 & -0.70406 & 0.02494 \\
xc & 17171.17928 & 3.18402 \\
w & 6622.42342 & 55.71842 \\
A & 17196.3629 & 345.64614
\end{tabular}




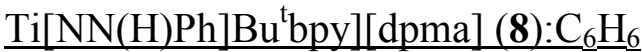

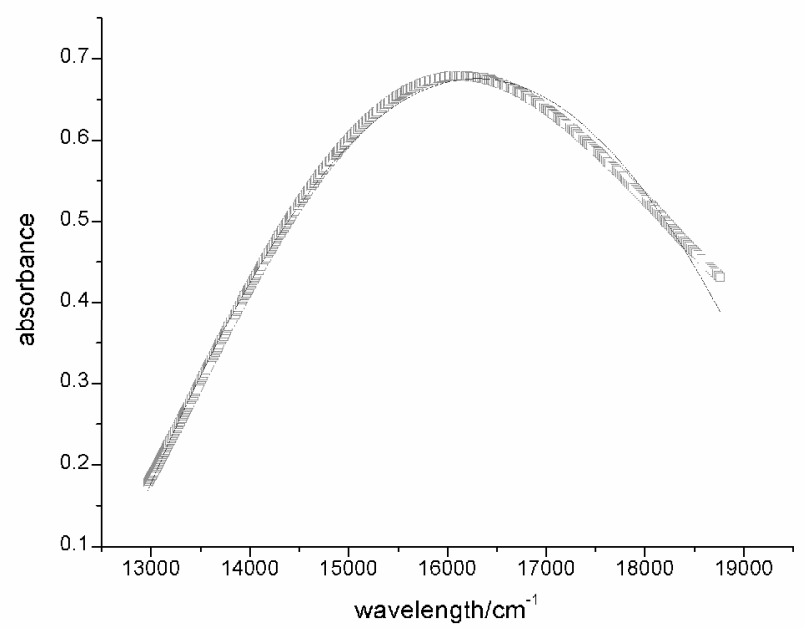

Data: $\operatorname{Ti}[\mathrm{NN}(\mathrm{H}) \mathrm{Ph}]\left[\mathrm{Bu}^{\mathrm{t}}\right.$ bpy] $[\mathrm{dpma}]: \mathrm{C}_{6} \mathrm{H}_{6}$

\section{Model: Gauss}

Equation: $\mathrm{y}=\mathrm{y}_{0}+\left(\mathrm{A} /\left(\mathrm{w}^{*}(\pi / 2)^{1 / 2}\right)\right) * \exp \left(-2 *\left(\left(\mathrm{x}-\mathrm{x}_{\mathrm{c}}\right) / \mathrm{w}\right)^{2}\right)$

Weighting:

y No weighting

$\mathrm{Chi}^{\wedge} 2 / \mathrm{DoF} \quad=0.0001$

$\mathrm{R}^{\wedge} 2=0.99523$

$\begin{array}{lll} & & \text { Error } \\ \text { y0 } & -2.79974 & 0.50894 \\ \text { xc } & 16300.19462 & 3.81786 \\ \text { w } & 11861.23275 & 924.53088 \\ \text { A } & 51657.40953 & 11584.1359\end{array}$




\section{$\underline{\left.\operatorname{Ti}[\mathrm{NN}(\mathrm{H}) \mathrm{Ph}] \mathrm{Bu}^{\mathrm{t} b p y}\right][\mathrm{dpma}](\mathbf{8}): \mathrm{THF}}$}

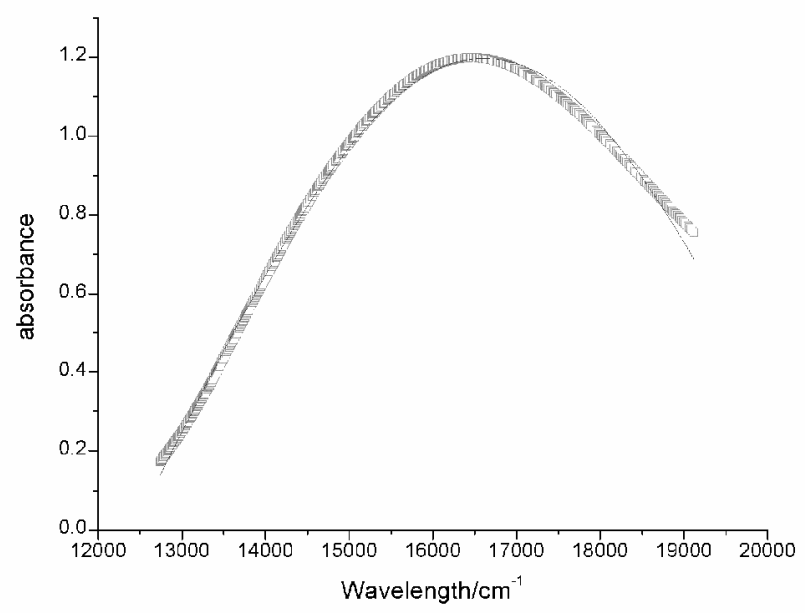

Data: $\mathrm{Ti}[\mathrm{NN}(\mathrm{H}) \mathrm{Ph}]\left[\mathrm{Bu}^{\mathrm{t}}\right.$ bpy] $[\mathrm{dpma}]: \mathrm{THF}$

Model: Gauss

Equation: $\mathrm{y}=\mathrm{y}_{0}+\left(\mathrm{A} /\left(\mathrm{w}^{*}(\pi / 2)^{1 / 2}\right)\right) * \exp \left(-2 *\left(\left(\mathrm{x}-\mathrm{x}_{\mathrm{c}}\right) / \mathrm{w}\right)^{2}\right)$

Weighting:

y No weighting

$\mathrm{Chi}^{\wedge} 2 / \mathrm{DoF} \quad=0.00028$

$\mathrm{R}^{\wedge} 2=0.9972$

$\begin{array}{lll} & & \text { Error } \\ \text { y0 } & -1.39381 & 0.07376 \\ \text { xc } & 16615.84957 & 3.69611 \\ \text { w } & 7569.24449 & 133.00815 \\ \text { A } & 24572.18134 & 1122.93346\end{array}$




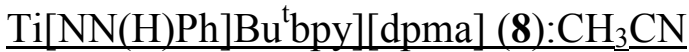

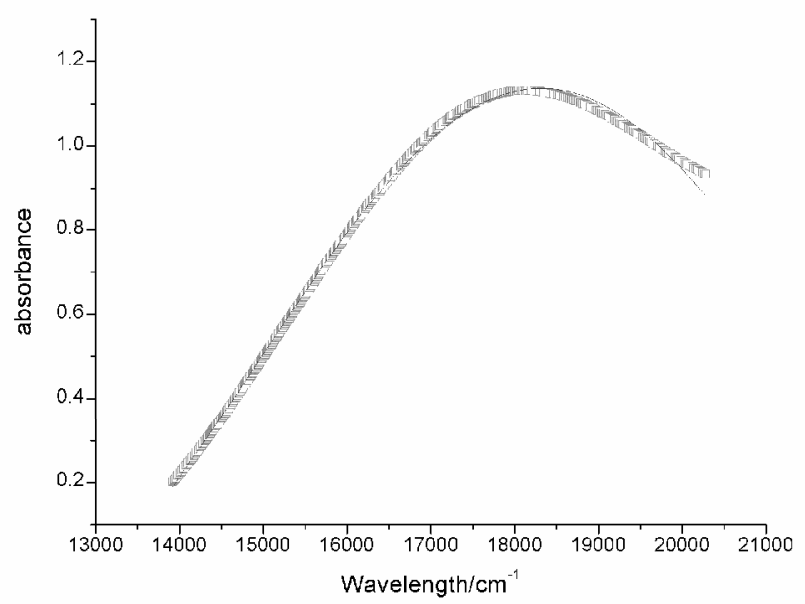

Data: $\operatorname{Ti}[\mathrm{NN}(\mathrm{H}) \mathrm{Ph}]\left[\mathrm{Bu}^{\mathrm{t}} \mathrm{bpy}\right][\mathrm{dpma}]$ :acetonitrile

Model: Gauss

Equation: $\mathrm{y}=\mathrm{y}_{0}+\left(\mathrm{A} /\left(\mathrm{w}^{*}(\pi / 2)^{1 / 2}\right)\right)^{*} \exp \left(-2^{*}\left(\left(\mathrm{x}-\mathrm{x}_{\mathrm{c}}\right) / \mathrm{w}\right)^{2}\right)$

Weighting:

y No weighting

$\mathrm{Chi}^{\wedge}$ 2/DoF $=0.00013$

$\mathrm{R}^{\wedge} 2=0.99861$

$\begin{array}{lll} & & \text { Error } \\ \text { y0 } & -0.56748 & 0.02349 \\ \text { xc } & 18320.95306 & 4.68298 \\ \text { w } & 6899.24324 & 68.76452 \\ \text { A } & 14729.40468 & 343.87654\end{array}$




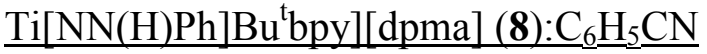

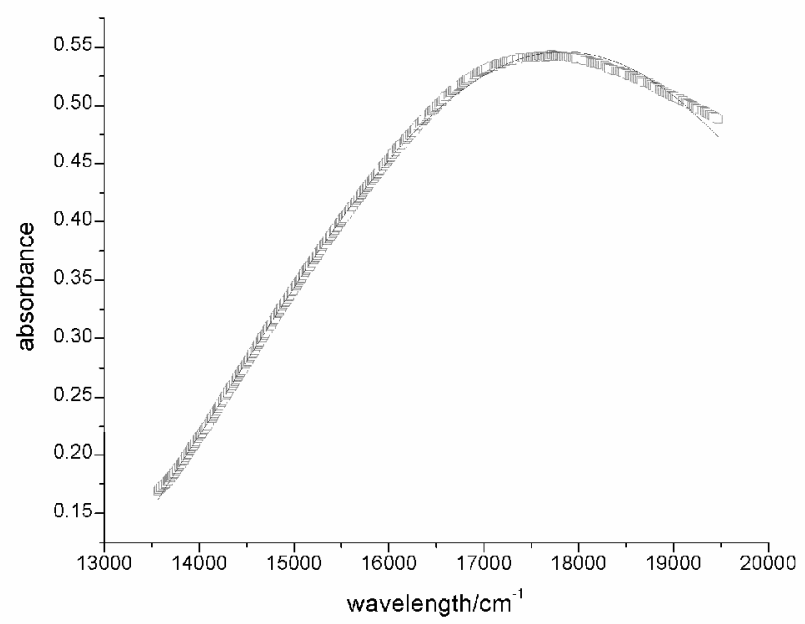

Data: $\operatorname{Ti}[\mathrm{NN}(\mathrm{H}) \mathrm{Ph}]\left[\mathrm{Bu}^{\mathrm{t}}\right.$ bpy] $[\mathrm{dpma}]$ :benzonitrile

Model: Gauss

Equation: $\mathrm{y}=\mathrm{y}_{0}+\left(\mathrm{A} /\left(\mathrm{w}^{*}(\pi / 2)^{1 / 2}\right)\right) * \exp \left(-2 *\left(\left(\mathrm{x}-\mathrm{x}_{\mathrm{c}}\right) / \mathrm{w}\right)^{2}\right)$

Weighting:

y No weighting

$\mathrm{Chi}^{\wedge} 2 / \mathrm{DoF} \quad=0.00001$

$\mathrm{R}^{\wedge} 2=0.99908$

$\begin{array}{lll} & & \text { Error } \\ \text { y0 } & -0.22694 & 0.0113 \\ \text { xc } & 17844.83341 & 4.68155 \\ \text { w } & 7297.13119 & 74.78863 \\ \text { A } & 7065.79562 & 173.70683\end{array}$




\section{Tables of $\Delta \mathbf{E}^{0}$ calculations for complexes 5-8}

Calculating $\Delta v_{1 / 2}$ after one Gaussian fit from the experimental UV-Vis plots of the complexes and plugging it in the following equation we get the information shown below

$\Delta v_{1 / 2}=\left[16(\ln 2) k_{\mathrm{B}} \mathrm{T}\left(\mathrm{E}_{\mathrm{op}}-\Delta \mathrm{E}^{\mathrm{o}}\right)\right]^{1 / 2}$ where $\mathrm{T}=293 \mathrm{~K} ; k_{\mathrm{B}}=0.69503 \mathrm{~cm}^{-1} \mathrm{~K}^{-1}$

$\operatorname{Ti}[\mathrm{NN}(\mathrm{Me}) \mathrm{Ph}][\mathrm{dpma}]\left[\mathrm{Bu}^{\mathrm{t}} \mathrm{bpy}\right](\mathbf{5})$

\begin{tabular}{|c|c|c|c|}
\hline $\begin{array}{c}\text { Ti[NNMePh] }\left[\mathrm{Bu}^{\mathrm{t}}-\right. \\
\text { bpy][dpma] }(\mathbf{5})\end{array}$ & $\Delta v_{1 / 2} / \mathrm{cm}^{-1}$ & $\mathrm{E}_{\mathrm{op}} / \mathrm{cm}^{-1}$ & $\Delta \mathrm{E}^{\mathbf{O}} / \mathrm{cm}^{-1}$ \\
\hline Benzonitrile & 5268 & 17067 & 4776 \\
\hline Acetonitrile & 5332 & 17775 & 5184 \\
\hline THF & 4870 & 16450 & 5945 \\
\hline Benzene & 4488 & 15288 & 6367 \\
\hline
\end{tabular}

$\operatorname{Ti}\left[\mathrm{NN}(\mathrm{Me})\left(4-\mathrm{CH}_{3} \mathrm{C}_{6} \mathrm{H}_{4}\right)\right]\left[\mathrm{Bu}^{\mathrm{t}}-\mathrm{bpy}\right][\mathrm{dpma}](\mathbf{6})$

\begin{tabular}{|c|c|c|c|}
\hline $\begin{array}{c}\text { Ti[NN(Me)(4- } \\
\begin{array}{c}\left.\left.\mathrm{CH}_{3} \mathrm{C}_{6} \mathrm{H}_{4}\right)\right]\left[\mathrm{Bu}^{\mathrm{t}}-\right. \\
\text { bpy][dpma] }](6)\end{array}\end{array}$ & $\Delta v_{1 / 2} / \mathrm{cm}^{-1}$ & $\mathrm{E}_{\mathrm{op}} / \mathrm{cm}^{-1}$ & $\Delta \mathrm{E}^{\mathrm{o}} / \mathrm{cm}^{-1}$ \\
\hline Benzonitrile & 5028 & 16755 & 5558 \\
\hline Acetonitrile & 5240 & 17237 & 5076 \\
\hline THF & 4550 & 16093 & 6924 \\
\hline Benzene & 4230 & 14842 & 6917 \\
\hline
\end{tabular}

$\operatorname{Ti}\left[\mathrm{NN}(\mathrm{Me})\left(4-\mathrm{FC}_{6} \mathrm{H}_{4}\right)\right]\left[\mathrm{Bu}^{\mathrm{t}}-\mathrm{bpy}\right][\mathrm{dpma}](7)$

\begin{tabular}{|c|c|c|c|}
\hline $\begin{array}{c}\mathrm{Ti}[\mathrm{NN}(\mathrm{Me})(4- \\
\left.\left.\mathrm{FC}_{6} \mathrm{H}_{4}\right)\right]\left[\mathrm{Bu}^{\mathrm{t}}-\right. \\
\text { bpy][dpma](7) }\end{array}$ & $\Delta v_{1 / 2} / \mathrm{cm}^{-1}$ & $\mathrm{E}_{\mathrm{op}} / \mathrm{cm}^{-1}$ & $\Delta \mathrm{E}^{\mathrm{o}} / \mathrm{cm}^{-1}$ \\
\hline Benzonitrile & 5218 & 17171 & 5112 \\
\hline Acetonitrile & 5614 & 17864 & 3905 \\
\hline THF & 4702 & 16552 & 6760 \\
\hline Benzene & 4486 & 15380 & 6467 \\
\hline
\end{tabular}

$\operatorname{Ti}[\mathrm{NN}(\mathrm{H}) \mathrm{Ph}]\left[\mathrm{Bu}^{\mathrm{t}}-\mathrm{bpy}\right][\mathrm{dpma}](\mathbf{8})$

\begin{tabular}{|c|c|c|c|}
\hline $\begin{array}{c}\mathrm{Ti}[\mathrm{NN}(\mathrm{H}) \mathrm{Ph}]\left[\mathrm{Bu}^{\mathrm{t}}-\right. \\
\text { bpy][dpma]}](8)\end{array}$ & $\Delta v_{1 / 2} / \mathrm{cm}^{-1}$ & $\mathrm{E}_{\mathrm{op}} / \mathrm{cm}^{-1}$ & $\Delta \mathrm{E}^{\mathrm{O}} / \mathrm{cm}^{-1}$ \\
\hline Benzonitrile & 5534 & 17845 & 4281 \\
\hline Acetonitrile & 5908 & 18321 & 2861 \\
\hline THF & 5222 & 16616 & 4539 \\
\hline Benzene & 4920 & 16300 & 5579 \\
\hline
\end{tabular}




\section{Plots of $E_{o p}-\Delta E^{\circ}$ versus $1 / D_{o p}-1 / D_{s}$ for complexes 6-8}

The plots were made using KaleidaGraph version 4.02 software using the equation

$$
\mathrm{y}=\mathrm{m} 1+\mathrm{m} 2 * \mathrm{M} 0
$$

where $\mathrm{y}$ is $\left(\mathrm{E}_{\mathrm{op}}-\Delta \mathrm{E}^{\circ}\right), \mathrm{M} 0$ is the value of $1 / \mathrm{n}^{2}-1 / \mathrm{D}_{\mathrm{s}}, \mathrm{m} 1$ is the intercept and $\mathrm{m} 2$ being the slope.

$\operatorname{Ti}\left[\mathrm{NN}(\mathrm{Me})\left(4-\mathrm{CH}_{3} \mathrm{C}_{6} \mathrm{H}_{4}\right)\right][\mathrm{dpma}]\left[\mathrm{Bu}^{\mathrm{t}} \mathrm{bpy}\right](\mathbf{6})$

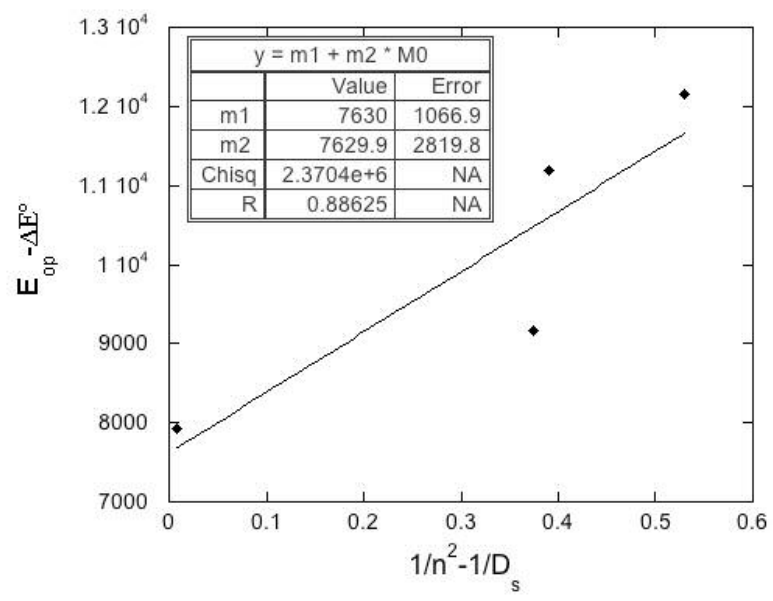

$\operatorname{Ti}\left[\mathrm{NN}(\mathrm{Me})\left(4-\mathrm{FC}_{6} \mathrm{H}_{4}\right)\right][\mathrm{dpma}]\left[\mathrm{Bu}^{\mathrm{t} b p y}\right](7)$

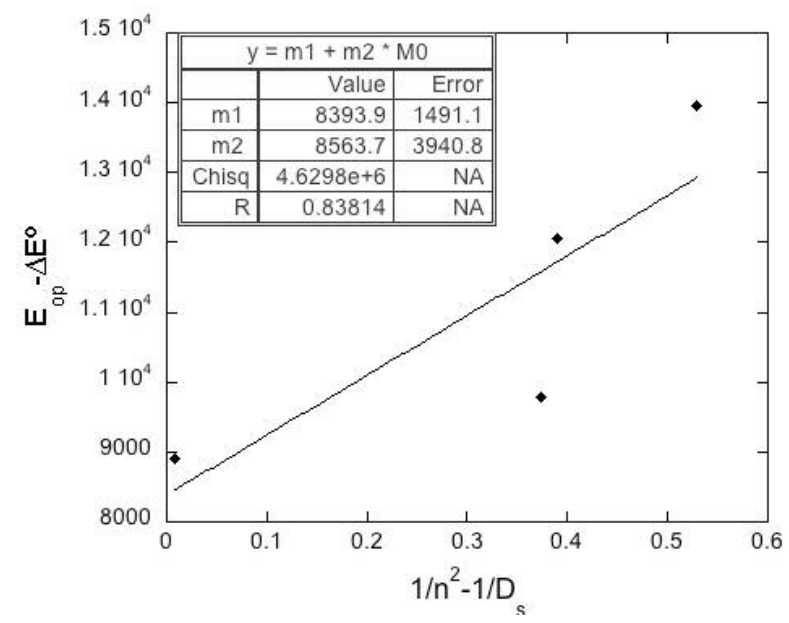


$\operatorname{Ti}[\mathrm{NN}(\mathrm{H}) \mathrm{Ph}][\mathrm{dpma}]\left[\mathrm{Bu}^{\mathrm{t}} \mathrm{bpy}\right](\mathbf{8})$

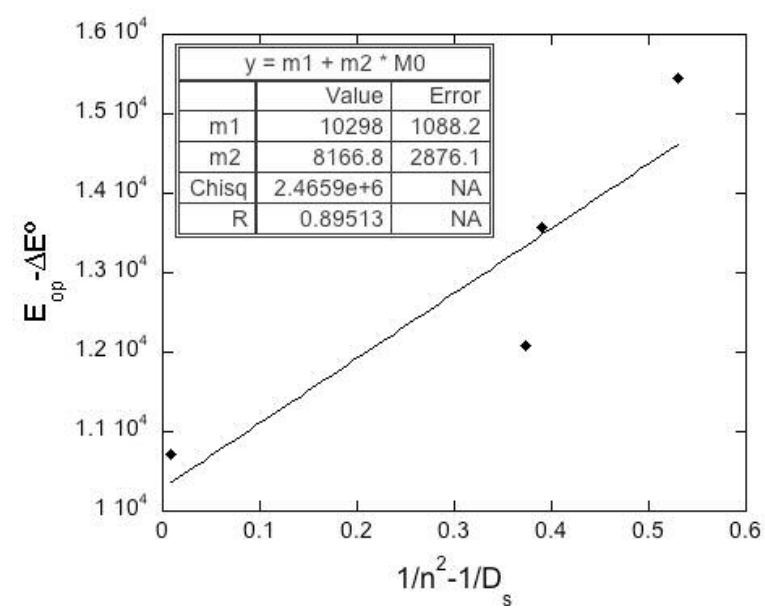


E. Solvent dielectric constant Vs $\mathrm{E}_{\text {op }}$ plots for complexes 2-8

$\operatorname{Ti}\left[\mathrm{NNMe}_{2}\right][\mathrm{Me}-\mathrm{bpy}][\mathrm{dpma}]$ (2)

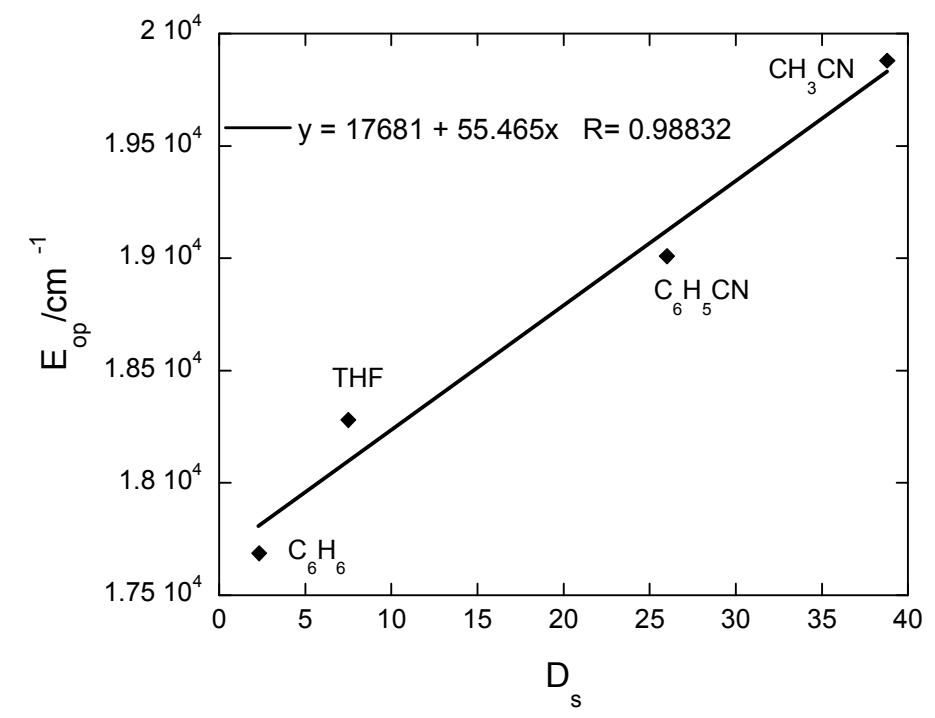

$\mathrm{y}=17681+55.465 \mathrm{x}$

$\mathrm{R}^{2}=0.988$

$\operatorname{Ti}\left[\mathrm{NNMe}_{2}\right][\mathrm{bpy}][\mathrm{dpma}](\mathbf{3})$

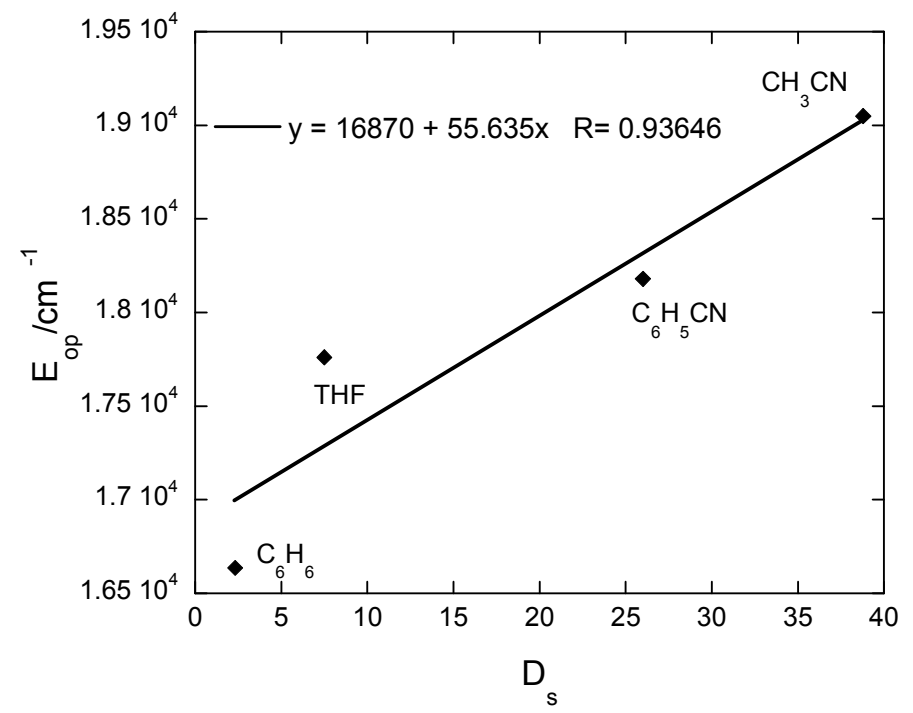

$\mathrm{y}=16870+55.635 \mathrm{x}$

$\mathrm{R}^{2}=0.936$ 
$\mathrm{Ti}\left[\mathrm{NNMe}_{2}\right][\mathrm{Ph}-\mathrm{bpy}][\mathrm{dpma}](\mathbf{4})$

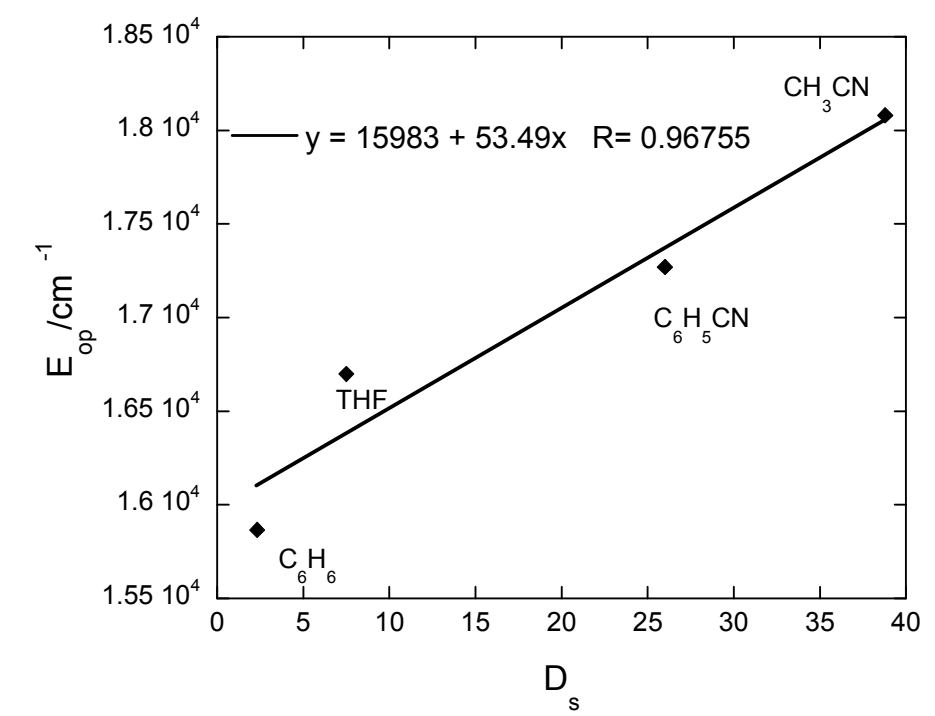

$\mathrm{y}=15983+53.49 \mathrm{x}$

$\mathrm{R}^{2}=0.968$

$\operatorname{Ti}[\mathrm{NN}(\mathrm{Me}) \mathrm{Ph}]\left[\mathrm{Bu}^{\mathrm{t}}-\mathrm{bpy}\right][\mathrm{dpma}](\mathbf{5})$

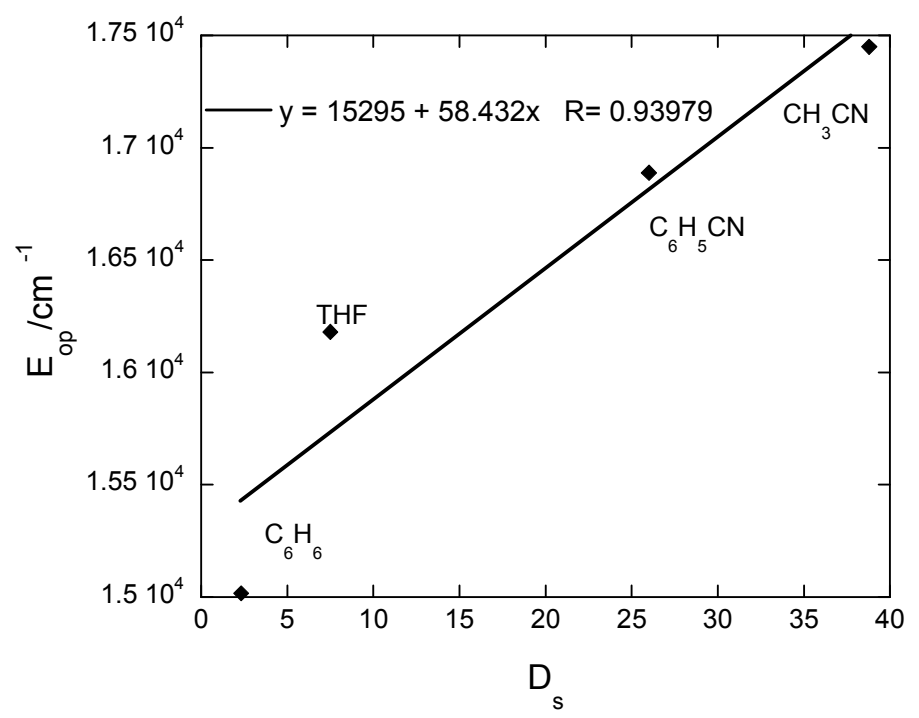

$\mathrm{y}=15295+58.43 \mathrm{x}$

$\mathrm{R}^{2}=0.940$ 
$\operatorname{Ti}[\mathrm{NN}(\mathrm{Me})(p$-tolyl $)]\left[\mathrm{Bu}^{\mathrm{t}}\right.$-bpy][dpma $](\mathbf{6})$

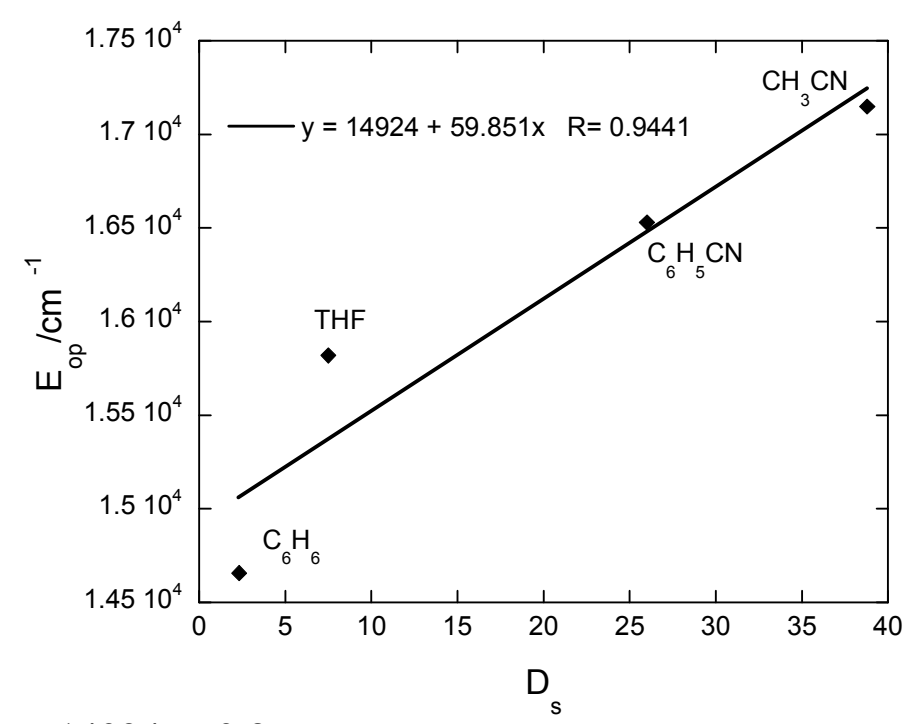

$\mathrm{y}=14924+59.85 \mathrm{x}$

$\mathrm{R}^{2}=0.944$

\section{$\operatorname{Ti}\left[\mathrm{NN}(\mathrm{Me})\left(p-\mathrm{FC}_{6} \mathrm{H}_{4}\right)\right]\left[\mathrm{Bu}^{\mathrm{t}}-\mathrm{bpy}\right][\mathrm{dpma}](7)$}

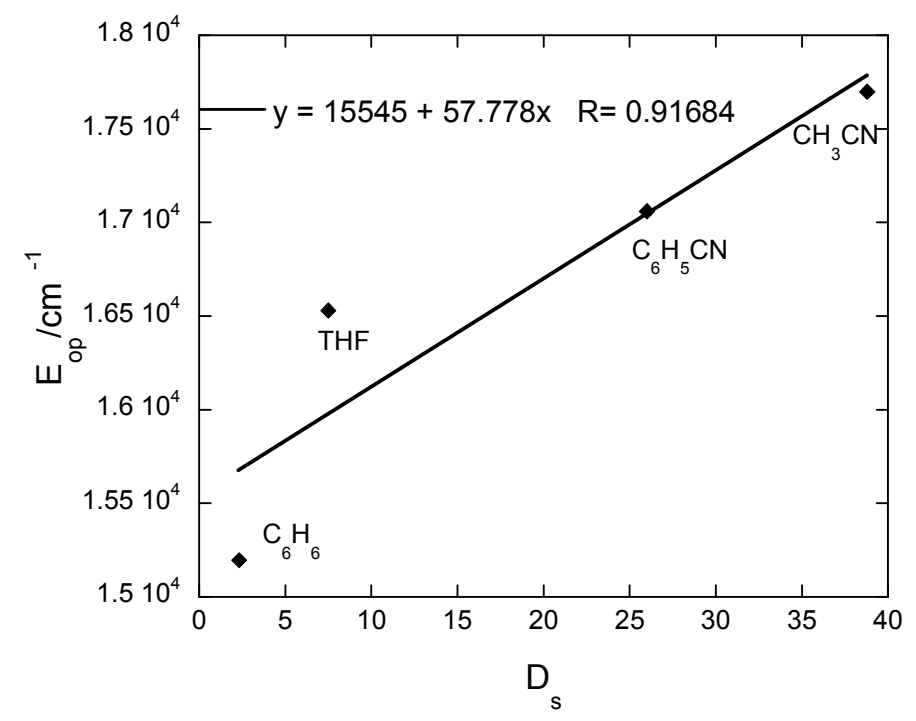

$y=15545+57.78 x$ 
$\mathrm{R}^{2}=0.917$

$\operatorname{Ti}[\mathrm{NN}(\mathrm{H}) \mathrm{Ph}]\left[\mathrm{Bu}^{\mathrm{t}}-\mathrm{bpy}\right][\mathrm{dpma}](\mathbf{8})$

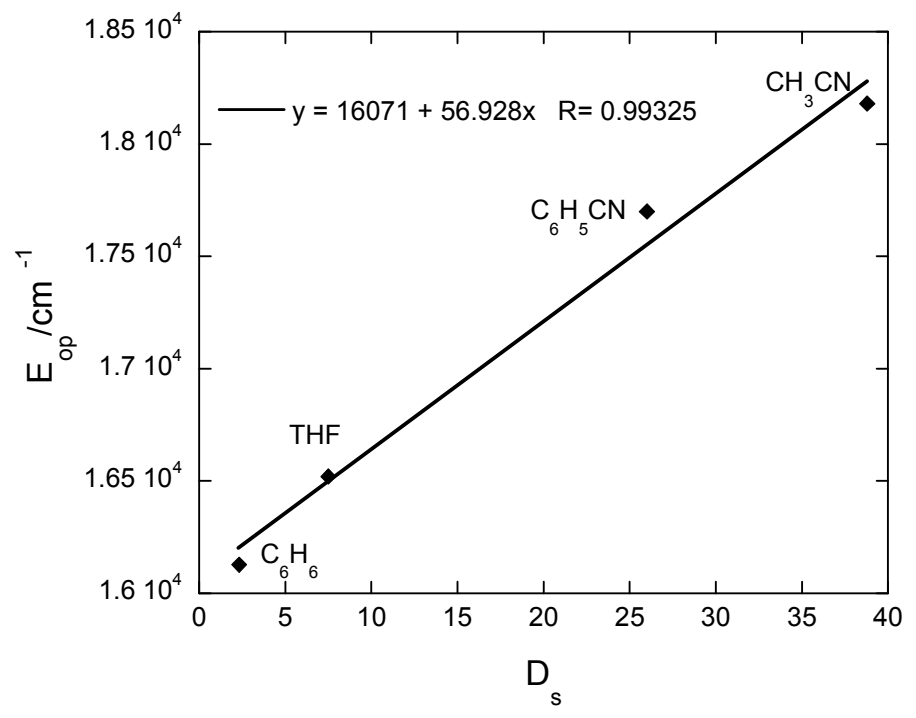

$\mathrm{y}=16071+56.93 \mathrm{x}$

$\mathrm{R}^{2}=0.993$ 
F. ${ }^{1} \mathrm{H}$ NMR spectrum for $\mathrm{Ti}\left[\mathrm{NN}(\mathrm{Me})\left(\mathrm{C}_{6} \mathrm{H}_{4}-p-\mathrm{F}\right)\right](\mathrm{dpma})\left(\mathrm{Bu}^{\mathrm{t}}-\mathrm{bpy}\right)(7)$

SP-III-15-3-1H-CDe13
Pi[KSHPS] (4paa] [Butbpy]

Pi[KSIPS] [ (apaa] [Butzpy

pulse Sequence: 82 pol

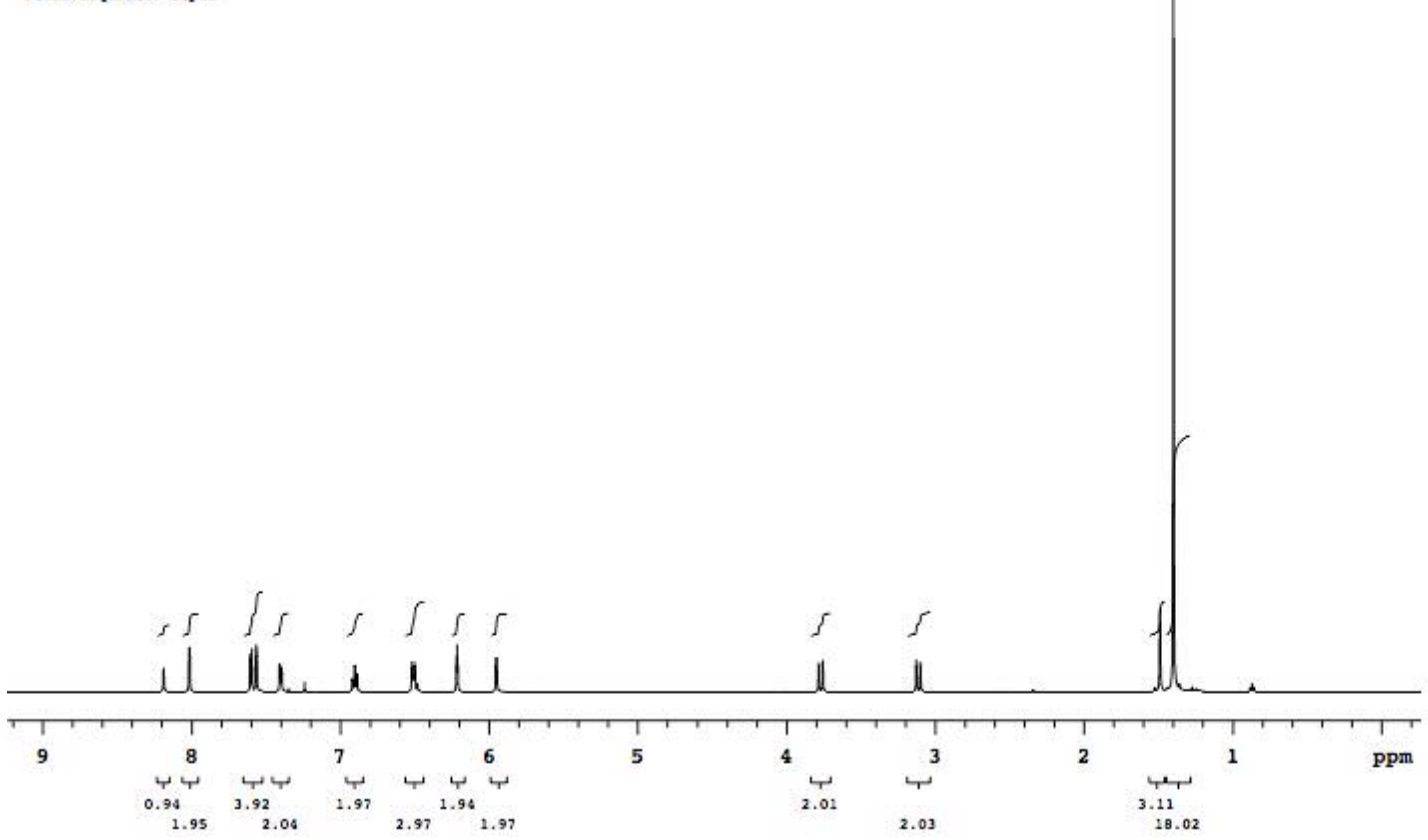

G. ${ }^{1}$ H NMR spectrum for $\mathrm{Ti}[\mathrm{NN}(\mathrm{H}) \mathrm{Ph}](\mathrm{dpma})\left(\mathrm{Bu}^{\mathrm{t}}-\mathrm{bpy}\right)(8)$

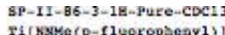
PI [NMMa (p-\{1eoropieay 1)] [Apea] [Butbpy

palke sequence: $52 \mathrm{pal}$

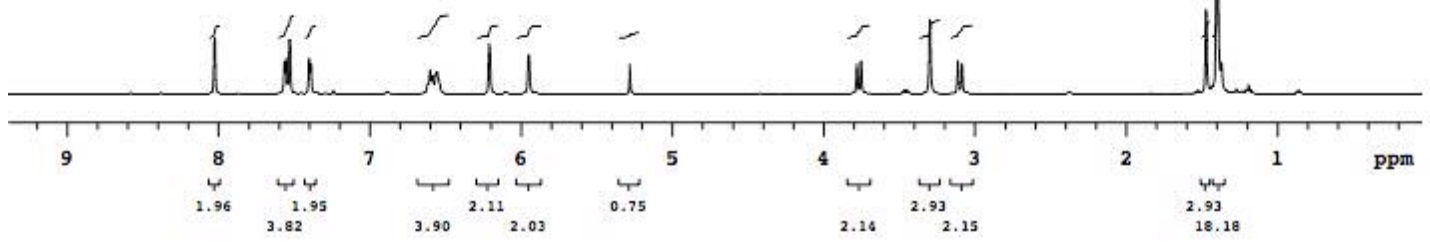

